\title{
Drift instability of standing Faraday waves
}

\author{
By ELENA MARTÍN. CARLOS MARTEL \\ AND JOSÉ M. VEGA \\ E. T. S. Ingenicros Industriales, Universidad de Vigo, Campus Lagoas, Marcosende, 9 , \\ 36200-Vigo, Pontevedra, Spain \\ E. T. S. Ingenicros Acronáuticos, Universidad Politécnica de Madrid, Plaza Cardenal Cisnoros, 3, \\ 28040-Madrid, Spain
}

We consider the weakly nonlinear evolution of the Faraday waves produced in a vertically vibrated two-dimensional liquid layer, at small viscosity. It is seen that the surface wave evolves to a drifting standing wave, namcly a wave that is standing in a moving reference frame. This wave is determined up to a spatial phase, whose calculation requires consideration of the associated mcan flow. This is just the streaming flow generated in the boundary layer attached to the lower plate supporting the liquid. A system of equations is derived for the coupled slow evolution of the spatial phase and the streaming flow. 'These equations are numerically integrated to show that the simplest reflection symmetric steady state (the usual array of counter-rotating eddies below the surface wave) becomes unstable for realistic values of the parameters. The new states include limit cycles (the array of eddies oscillating laterally), drifted standing waves (patterns that are standing in a uniformly propagating reference frame) and some more complex altractors.

\section{Introduction}

We consider the gravity-capillary waves named after Faraday (1831) that are parametrically excited at the free surface of a liquid when the container is vertically vibrated and the driven acceleration exceeds a threshold value. These waves have been receiving renewed and increasing attention in the literature owing in part to the impressive varicty of experimentally observed bchaviour (already reported by Faraday, see Miles \& I Ienderson 1990) that current theoretical approaches fail to explain convincingly, specially in the low-viscosity limit. This limit is singular and its analysis is subtle due in part to the presence of mean flows, which are well known to affect the surface wave dynamics in related systems (e.g. Milewski \& Benney 1995; Mashayek \& Ashgriz 1998) and are frequently cither ignored or treated in a deficient way. Strictly inviscid mean flows have already appeared in the celebrated equations derived by Davey \& Stewartson (1974), but these flows neglect the effect of viscous boundary layers, which can be essential even for quite small viscosity. These boundary layers produce a viscous streaming flow (also called steady streaming or acoustic strcaming), which has been extensively analysed in other contexts (Rilcy 2001 and references therein). Also, this flow (i) was indirectly observed by Faraday (1831) through the accumulation of sand at the bottom of the container, (ii) is sometimes used to visualize the patterns (Douady 1990) and (iii) has been associated with pattern rotation (Kiyashko et al. 1996); but it is otherwise ignored in connection with Faraday waves. 


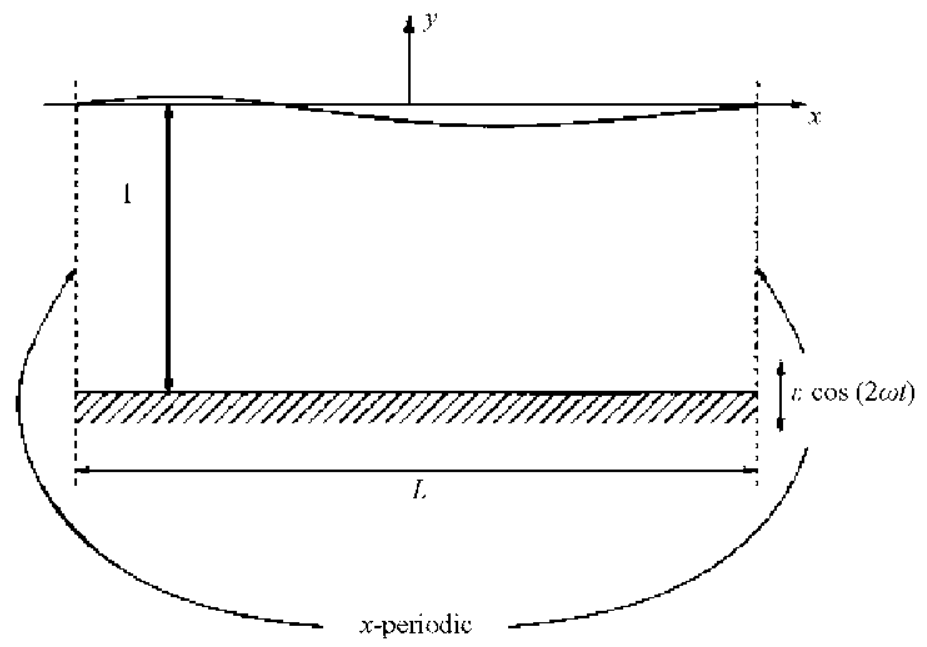

FIcicre: 1. Sketch of the two-dimensional annular domain.

The role of the streaming flow is better uncovered in the absence of additional effects like wave modulation, which leads to a complex description (Vega, Knobloch \& Martel 2001). Thus, we consider a spatially uniform surface wave, which seems to be the one involved in some of the drift modes encountered by Douady, Fauve \& 'Thual (1989) in annular domains. 'These consisted of a drifted standing wave, i.e. a spatially uniform, monochromatic wave that would be standing in a uniformly rotating reference frame; see also Thual, Douady \& Fauve (1989 p. 236) and Fauve, Douady \& Thual (1991 pp. 315-316) for a phenomenological description and Cross \& Hohenberg (1993 p. 1026) for a further discussion on these patterns.

The usual amplitude equations in the literature for weakly damped, spatially uniform, monochromatic Faraday waves in a domain that allows propagation in a particular direction account for damping, detuning, nonlinearity and parametric forcing, are

$$
\begin{aligned}
& A^{\prime}=\left[-\delta-\mathrm{i} d+\mathrm{i} \alpha_{3}|A|^{2}-\mathrm{i} \alpha_{4}|B|^{2}\right] A+\mathrm{i} \varepsilon \alpha_{5} \bar{B}, \\
& B^{\prime}=\left[-\delta-\mathrm{i} d+\mathrm{i} \alpha_{3}|B|^{2}-\mathrm{i} \alpha_{4}|A|^{2}\right] B+\mathrm{i} \varepsilon \alpha_{5} \bar{A},
\end{aligned}
$$

where $A$ and $B$ are the complex amplitudes of two counter-propagating waves and $|A| \sim|B| \ll 1, \delta \ll 1, d \ll 1$ and $\varepsilon \ll 1$, while the coefficients $\alpha_{3}, \alpha_{4}$ and $\alpha_{5}$ are of order unity. These equations ignore the streaming flow and lead to inconsistencics, as we show now. The large time behaviour of (1.1)-(1.2) are standing waves (SWs) of the form $|A|=|B|=$ constant, determined up to a spatial phase $w$, which can be defined as proportional to the difference between the phases of $A$ and $B$, and is given by

$$
\psi^{\prime}=0
$$

Note that $\psi^{\prime}$ is a drift velocity of the pattern. On the other hand, if the fluid domain is a two-dimensional laterally unbounded (figure 1), with periodic boundary conditions, then after appropriate rescaling the streaming flow produced by the SWs is given by the following well-known (sce, c.g. Iskandarani \& Liu 1991) system of cquations and boundary conditions

$$
\begin{gathered}
\tilde{u}_{x}+\tilde{v}_{y}=0, \\
\partial \tilde{u} / \partial \tau+\tilde{v}\left(\tilde{u}_{y}-\tilde{v}_{x}\right)=-\tilde{q}_{x}+\operatorname{Re}{ }^{1}\left(\tilde{u}_{x x}+\tilde{u}_{y y}\right),
\end{gathered}
$$




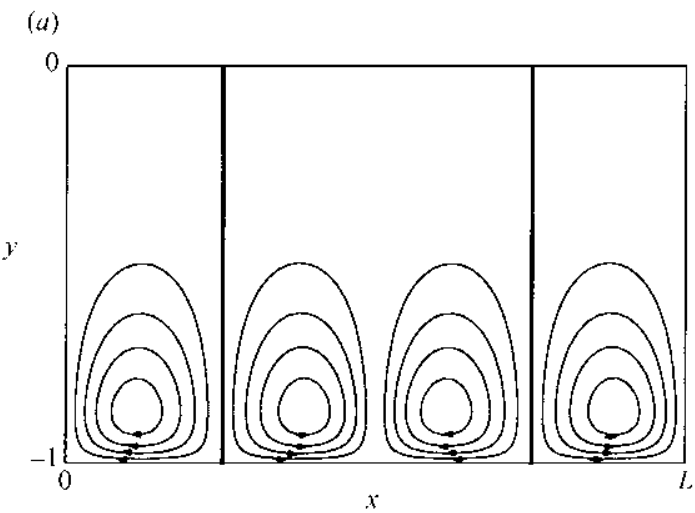

(b)

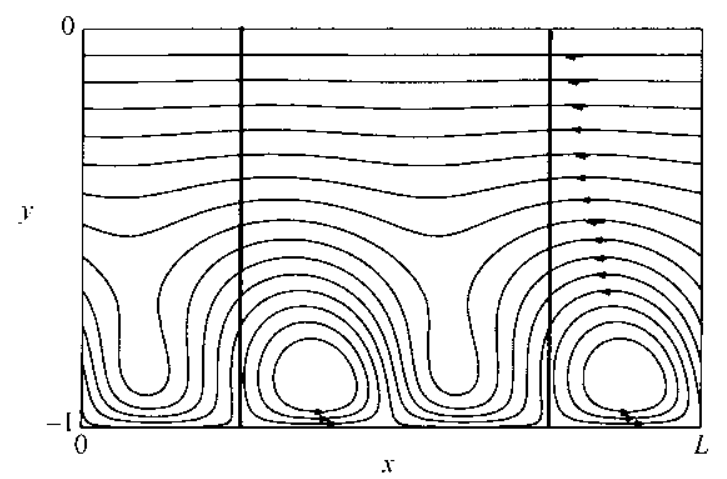

Figure 2. Streamlines of the stable steady state of (4.8)-(4.13), with $\psi=0$, for $L=\frac{1}{2} \pi, m=1(k=4)$. (a) $R e=55$, (b) $R e=70$. Thick vertical lines correspond to the nodes of the surface waves, given by $(4.23)$.

$$
\begin{gathered}
\hat{\partial v} / \partial \tau-\tilde{u}\left(\tilde{u}_{y}-\tilde{v}_{x}\right)=-\tilde{q}_{y}+\operatorname{Re}^{1}\left(\tilde{v}_{x x}+\tilde{v}_{y y}\right), \\
\tilde{u}=-\sin 2 k x, \quad \tilde{v}=0 \quad \text { at } \quad y=-1, \\
\partial \tilde{u} / \partial y=\tilde{v}=0, \quad \text { at } \quad y=0,
\end{gathered}
$$

$\tilde{u}, \tilde{v}$ and $\tilde{q}$ are $x$-periodic, of period $L=2 m \pi / k$.

The forcing term in (1.7) comes from the well-known slowly varying effect of the Stokes boundary layer attached to the lower plate (Batchelor 1967). The simplest solution to this problem is a stationary array of counter-rotating eddies, like that in figure 2(a), which has been numerically obtained for $L=\frac{1}{2} \pi, m=1(k=4)$ and $R e=55$. This pattern was qualitatively described by Liu \& Davis (1977) and numerically calculated by Iskandarani \& Liu (1991), and is always assumed in the literature (e.g. Douady 1990 figure 12) to be the only one associated with SWs. The pattern is $x$-reflection-symmetric; thus it cannot induce any drift of the waves, which is consistent with (1.3). However, this steady state of (1.4)-(1.9) is unstable if $R e$ exceeds a threshold value, which has not been noticed before to our knowledge. In fact, a direct numerical integration (1.4) (1.9) shows that the symmetric steady state is stable only below the dashed curve in the Re vs. $L$ diagram in figure 6 . At this curve, there is a symmetry breaking (pitchfork) bifurcation. The resulting stable, non-reflection-symmetric patterns for higher $R e$ are qualitatively similar to that in figure $2(b)$, and exhibit a non-zero overall horizontal velocity; see Yan, Ingham \& 
Morton (1993) for a similar result on a related problem. That streaming flow pattern must induce a drift of the primary SW by convection, namely a non-zero term in the right-hand side of (1.3). Thus, the usual amplitude equations (1.1)-(1.2) are faulty. The correct equations should give the coupled evolution of the phase $\psi$ and the streaming flow in a natural way. In order to avoid further deficiencics, we will not rely on any phenomenological argument. Instead, we shall derive the amplitude equations from first principles, though for the sake of brevity some ingredients of these cquations that are well-known will be omitted.

With these ideas in mind, the remainder of the paper is organized as follows. The problem is formulated in $\$ 2$. The correct extension of $(1.1)-(1.2)$ is derived in $\$ 3$ and used in $\$ 4.1$ first to show that the surface wave evolves to a drifting SW, namely, a wave that is standing in a moving reference frame, and then to obtain a system of equations giving the coupled evolution of the spatial phase $y$ and the streaming flow. This system of equations will be numerically explored in $\$ 4.2$, to obtain several steady and time-dependent solutions that exhibit a non-zero drift of the surface waves. Some conclusions will be made in $\$ 5$.

\section{Formulation}

We consider a horizontal two-dimensional liquid layer supported by a vertically vibrating plate (figure 1). We use the height of the unperturbed free surface $h$ and the gravitational time $(h / g)^{1 / 2}$ (where $g$ is the acceleration due to gravity) as characteristic length and time for non-dimensionalization. The governing equations are

$$
\begin{gathered}
u_{x}+v_{y}=0 \\
u_{t}+v\left(u_{y}-v_{x}\right)=-q_{x}+C\left(u_{x x}+u_{y y}\right), \\
v_{t}-u\left(u_{y}-v_{x}\right)=-q_{y}+C\left(v_{x x}+v_{y y}\right), \\
u=v=0 \text { at } y=-1, \\
v=f_{i}+u f_{x}, \quad\left(u_{y}+v_{x}\right)\left(1-f_{x}^{2}\right)+2\left(v_{y}-u_{x}\right) f_{x}=0 \text { at } y=f, \\
q-\left(u^{2}+v^{2}\right) / 2+4 \omega^{2} \varepsilon f \cos (2 \omega t)-f+T f_{x x} /\left(1+f_{x}^{2}\right)^{3 / 2} \\
=2 C\left[v_{y}+u_{x} f_{x}^{2}-\left(u_{y}+v_{x}\right) f_{x}\right] /\left(1+f_{x}^{2}\right) \text { at } y=f,
\end{gathered}
$$

$u, v, p$ and $f$ are periodic, of period $L$, in $x$.

For convenience, we also recall that volume is conserved, i.e.

$$
\int_{0}^{L} f(x, t) \mathrm{d} x=0 .
$$

Here, $u$ and $v$ are the horizontal and vertical velocity components, $f$ is the free surface elevation (measured from the undisturbed position), and $q=$ pressure + $\left(u^{2}+v^{2}\right) / 2+y-4 \omega^{2} \varepsilon y \cos (2 \omega t) ; 2 \omega$ and $\varepsilon$ are the forcing frequency and amplitude, $C=v /\left(g h^{3}\right)^{1 / 2}(v=$ kincmatic viscosity $)$ is a ratio of viscous to gravitational effects and $T^{-1}=\rho g h^{2} / \sigma(\rho=$ density, $\sigma=$ surface tension $)$ is the Bond number.

We shall consider small, nearly resonant solutions at small viscosity, i.e.

$$
|u|+|v|+|q|+|f| \ll 1, \quad \varepsilon \ll 1, \quad\left|\omega-\omega_{0}\right| \ll 1, \quad C \ll 1,
$$

without further restrictions. Here, $\omega_{0}$ is a natural frequency in the inviscid limit. 


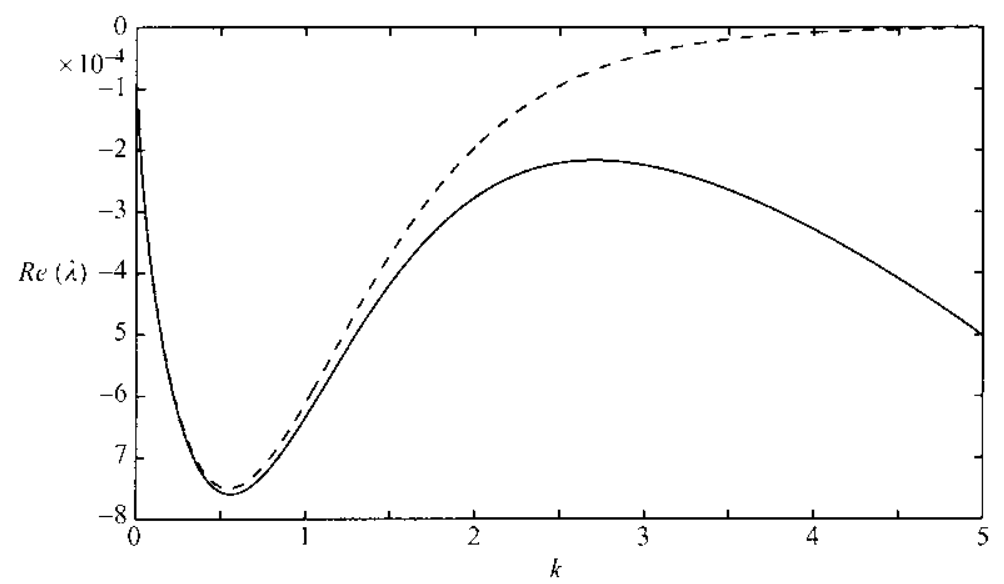

Figure 3. Damping rate of the surface modes for $C=10^{5}$ and $T=7.3 \times 10^{4}$ using ---, the $O\left(C^{1 / 2}\right)$ results and - the $O(C)$ results; the latter are indistinguishable from those obtained using the exact dispersion relation.

\section{Coupled amplitude-mean flow equations in two dimensions}

The weakly nonlinear analysis of (2.1)-(2.7) in the limit (2.9) requires consideration of all nearly marginal modes of the lincarization of $(2.1)-(2.7)$ around the quicscent state $(u, v, q, f)=(0,0,0,0)$ in the unforced case $\varepsilon=0$. $\Lambda s C \rightarrow 0$, the linearized problem exhibits two kind of nearly marginal mode.

Surface modes account for surface waves and are the only ones considered so far in weakly nonlinear theories. These modes exhibit a dispersion relation

$$
\lambda= \pm \mathrm{i}\left(\omega_{0}-(1 \pm \mathrm{i}) \alpha_{1} C^{1 / 2}-\alpha_{2} C+O\left(C^{3 / 2}\right)\right.
$$

where the inviscid eigenfrequency $\omega_{0}>0$ and the coefficients $\alpha_{1}>0$ and $\alpha_{2}>0$ depend on the wavenumber $k$ as

$$
\left.\begin{array}{c}
\omega_{0}^{2}=k\left(1+T k^{2}\right) \tanh k, \quad \alpha_{1}=k\left(\omega_{0} / 2\right)^{1 / 2} / \sinh 2 k \\
\alpha_{2}=k^{2}\left[2+\left(5+3 \tanh ^{2} k\right)\right] /\left(16 \sinh ^{2} k\right)
\end{array}\right\}
$$

Thus, these modes are oscillatory, with a small (as $C \rightarrow 0$ ) damping rate that results from viscous dissipation in the Stokes boundary layer at the bottom plate and in the bulk (essentially, the terms of orders $C^{1 / 2}$ and $C$, respectively); the neglected terms essentially come from viscous dissipation in the upper boundary layer at the free surface. The velocity jump across the lower boundary layer decays (and thus $\alpha_{1} \rightarrow 0$ ) exponentially as $k \rightarrow \infty$. Then, for a small but fixed value of $C$ and moderately large $k$ it is advisable to use the three-term expansion displayed in (3.1), which yields a good approximation for realistically small, fixed values of $C$ and $k$ in a wide range (Martel \& Knobloch 1997) as seen in figure 3, where the selected values of $C$ and $T$ correspond to water in a $10.1 \mathrm{~cm}$ deep container. Similar, three-term expansions are required for related two-dimensional and three-dimensional gravity-capillary wave problems in finite geometries (Higuera, Nicolás \& Vega 1994; Higuera \& Nicolás 1997; Martel, Nicolás \& Vega 1998).

Hydrodynamic modes (also called viscous modes) are those associated with the streaming flow and exhibit a dispersion relation (Lamb 1932)

$$
\lambda=-C\left[k^{2}+q_{n}(k)^{2}\right]+O\left(C^{2}\right) \text { at } C \rightarrow 0,
$$


where $q_{n}(k)$ are the solutions of $q_{n} \tanh k=k \tan q_{n}$. The associated eigenmodes yield no frec-surface deflection at leading order; thus, these modes can be ignored in a strictly linear theory if only the free-surface deflection matters, but not in other circumstances.

With these ideas in mind, let us now consider (2.1)-(2.7) in the limit (2.9). Outside these two boundary layers, we consider solutions of the form

$$
\left.\begin{array}{l}
u=U_{0}(y) \mathrm{e}^{\mathrm{i} \omega t}\left[A(t) \mathrm{e}^{\mathrm{i} k x}-B(t) \mathrm{e}^{-\mathrm{i} k x}\right]+\text { c.c. }+u^{s}(x, y, t)+\cdots, \\
v=V_{0}(y) \mathrm{e}^{\mathrm{i} k t h}\left[A(t) \mathrm{e}^{\mathrm{i} k x}+B(t) \mathrm{e}^{-\mathrm{i} k x}\right]+\text { c.c. }+v^{s}(x, y, t)+\cdots, \\
q=Q_{0}(y) \mathrm{e}^{\mathrm{i} \omega t}\left[A(t) \mathrm{e}^{\mathrm{i} k x}+B(t) \mathrm{e}{ }^{\mathrm{i} k x}\right]+\text { c.c. }+q^{s}(x, y, t)+\cdots, \\
f=\mathrm{e}^{\mathrm{i} \omega t}\left[A(t) \mathrm{e}^{\mathrm{i} k x}+B(t) \mathrm{e}^{-\mathrm{i} k x}\right]+\text { c.c. }+f^{s}(x, t)+\cdots,
\end{array}\right\}
$$

where c.c. stands for the complex conjugate and the horizontal wavenumber and the inviscid eigenfunctions $U_{0}, V_{0}$ and $Q_{0}$ are

$$
\begin{gathered}
k=2 m \pi / L \quad \text { with } \quad m=\text { integer, } \\
U_{0}=-k Q_{0} / \omega_{0}, \quad V_{0}=\mathrm{i} Q_{0 y} / \omega_{0}, \quad Q_{0}=\omega_{0}^{2} \cosh [k(y+1)] /[k \sinh k\rceil,
\end{gathered}
$$

in terms of the inviscid eigenfrequency $\omega_{0}$, which is related to the wavenumber $k$ and the forcing frequency $2 \omega$ by (3.2) and (2.9), respectively. In (3.4) we have displayed only the leading order (in the limit (2.9)) oscillatory and non-oscillatory terms, the latter being associated with the streaming flow and denoted hereinafter by the superscript s. The former correspond to the only surface mode that is (subharmonically) excited by the external forcing; the remaining non-excited surface modes are readily seen to decay cxponcntially in the time scalc associatcd with viscous dissipation and can be ignored. The weakly nonlinear level of our description requires that the complex amplitudes and the remaining slowly varying quantities be small and depend weakly on timc, i.c.

$$
\begin{gathered}
\left|A^{\prime}\right| \ll|A| \ll 1, \quad\left|B^{\prime}\right| \ll|B| \ll 1, \quad\left|u_{t}^{s}\right| \ll\left|u^{s}\right| \ll 1, \quad\left|v_{t}^{s}\right| \ll\left|v^{s}\right| \ll 1, \\
\left|q_{t}^{s}\right| \ll\left|q^{s}\right| \ll 1, \quad\left|f_{t}^{s}\right| \ll\left|f^{s}\right| \ll 1 .
\end{gathered}
$$

\subsection{The slow evolution of the complex amplitudes $A$ and $B$}

In order to obtain the amplitude equations giving $A$ and $B$, we could proceed in a standard manner, as follows. Add higher-order terms (proportional to powers of the small quantities $C^{1 / 2}, \varepsilon, A, B, u^{s}, v^{s}, q^{s}$ and $f^{s}$ ) to the expansions (3.4), and introduce similar expansions for $A^{\prime}$ and $B^{\prime}$. When these cxpansions are inserted into (2.1)-(2.3), and the coefficient of each asymptotic order is set to zero, a recurrent system of equations in the bulk is obtained; the appropriate boundary conditions at $y=-1$ and 0 are obtained by a matching procedure with the solutions in the lower and upper boundary layers, which must be analysed separately. Then, a solvability condition applicd to each of these problems provides the subsequent terms in the expansions for $A^{\prime}$ and $B^{\prime}$, which (to the approximation relevant here) are

$$
\begin{aligned}
& A^{\prime}=\left[-\delta-\mathrm{i} d+\mathrm{i} \alpha_{3}|A|^{2}-\mathrm{i} \alpha_{4}|B|^{2}-\mathrm{i} \alpha_{6} L^{-1} \int_{-1}^{0} \int_{0}^{L} g(y) u^{s} \mathrm{~d} x \mathrm{~d} y\right] A+\mathrm{i} \varepsilon \alpha_{5} B, \\
& B^{\prime}=\left[-\delta-\mathrm{i} d+\mathrm{i} \alpha_{3}|B|^{2}-\mathrm{i} \alpha_{4}|A|^{2}+\mathrm{i} \alpha_{6} L^{-1} \int_{-1}^{0} \int_{0}^{l} g(y) u^{s} \mathrm{~d} x \mathrm{~d} y\right] B+\mathrm{i} \varepsilon \alpha_{5} \bar{A},
\end{aligned}
$$


where the parameters

$$
\delta=\alpha_{1} C^{1 / 2}+\alpha_{2} C, \quad d=\alpha_{1} C^{1 / 2}+\omega_{0}-\omega,
$$

are small (see (2.9)) and account for linear damping and detuning, respectively.

Now, the above procedure (which is quite tedious) can be avoided if proceeding as follows. 'The cocflicients associated with lincar damping and detuning, $\delta$ and $d$, can be taken from the dispersion relation (3.1). The remaining terms on the right-hand side of (3.7) (3.8) do not depend explicitly on $C$ and coincide with their counterparts in the usual amplitude equations in the literature (Miles 1993; Milner 1991; Hansen \& Alstrom 1997). For completeness, these and the function $g$ are calculated in the $\Lambda$ ppendix to be

$$
\begin{gathered}
\alpha_{3}=2 \omega_{0} k^{2}+\frac{\omega_{0} k^{2}}{4 \sigma^{2}} \frac{\left(9-\sigma^{2}\right)\left(1-\sigma^{2}\right)+\left(7-\sigma^{2}\right)\left(3-\sigma^{2}\right) T k^{2}}{\sigma^{2}+\left(\sigma^{2}-3\right) T k^{2}}-\frac{3 T \omega_{0} k^{4}}{4\left(1+T k^{2}\right)}, \\
\alpha_{4}=\frac{\omega_{0} k^{2}}{2}\left[\frac{\left(\sigma^{2}+1\right)^{2}}{\sigma^{2}} \frac{1+T k^{2}}{1+4 T k^{2}}+\frac{4+7 T k^{2}}{1+T k^{2}}\right], \quad \alpha_{5}=\omega_{0} k \sigma, \\
\alpha_{6}=\frac{k \sigma}{2 \omega_{0}}, \quad g(y)=\frac{2 \omega_{0} k \cosh [2 k(y+1)]}{\sinh ^{2} k},
\end{gathered}
$$

where the eigenfrequency $\omega_{0}$ is as given by (3.2) and $\sigma=\tanh k$.

Some remarks about the amplitude equations (3.7)-(3.8) are now in order.

(i) Some care must be taken with that calculation because the limit $C \rightarrow 0$ is a singular perturbation limit and does not necessarily commute with the limit $|A|+|B| \rightarrow 0$. However, a careful analysis of the oscillatory boundary layers (as that in Nicolás \& Vega 1996) shows that the limits $C \rightarrow 0$ and $|A|+|B| \rightarrow 0$ do commute as far as the calculation of those terms that are independent of $C$ on the right-hand sides of (3.7)-(3.8) is concerned.

(ii) The amplitude equations are invariant under the actions $A \rightarrow A \mathrm{e}^{\mathrm{ic}}, B \rightarrow B \mathrm{e}$ ic (for all c) and $A \leftrightarrow B, x \rightarrow-x, u^{v} \rightarrow-u^{v}$, which result from the invariance of (2.1)-(2.7) under $x$-translations and left-right $x$-reflection. Thus, only one of these equations need be derived.

(iii) We are not retaining the real parts of the coefficients of the cubic terms in (3.7) (3.8), which account for nonlinear damping and forcing and would be necessary only near the instability threshold if detuning is appropriatcly small.

(iv) Since $\left|u^{s}\right|$ will turn out to be of the order of $|A|^{2}+|B|^{2}$, the new integral terms in (3.7)-(3.8) are of the same order as that of the cubic terms and cannot be ignored. Note that the new terms are conservative, namely they do not contribute to the leading-order energy equation obtained by multiplying (3.7) and (3.8) by the complex conjugates of $A$ and $B$, adding and taking the real part. This is consistent with the fact that the streaming flow velocity is small compared to the velocity associated with the surface waves and thus it does not contribute to the kinetic energy at leading order.

The slowly varying parts of the velocity components, $u^{s}$ and $v^{s}$, remain undetermined at this stage. The slowly varying parts of momentum equations at leading order (just give $q_{x}^{s}=q_{y}^{s}=0$, and) do not involve $u^{s}$ and $v^{s}$, which are usually set to zero in current Faraday wave theories invoking continuity and imposing zero vorticity. The latter would be justified in the strictly inviscid case (if, in addition, the flow is initially potential), but, for non-zero viscosity, vorticity is necessarily present in the oscillatory boundary layers produced by the surface waves, and it can (and will) be convected 
and/or diffused to the bulk to yield a non-zero vortical flow, which is precisely the strcaming flow.

\subsection{The streaming flow}

The slowly varying parts of the velocity components, $u^{s}$ and $v^{s}$, are now calculated from the continuity equation at order $\left|u^{s}\right|+\left|v^{s}\right|$ and the momentum equations at order $\left(|A|^{2}+|B|^{2}\right)\left(\left|u^{s}\right|+\left|v^{s}\right|\right)$. These lead to

$$
\begin{gathered}
u_{x}^{s}+v_{x}^{s}=0, \\
u_{t}^{s}+v^{s}\left(u_{y}^{s}-v_{x}^{s}\right)+q_{x}^{s}-C\left(u_{x x}^{s}+v_{y y}^{s}\right)=V_{0}(y)\left(A \mathrm{e}^{\mathrm{i} k x}+B \mathrm{e}^{-i k x}\right)\left(\bar{V}_{x}^{3}-\bar{U}_{y}^{3}\right)+\text { c.c. }, \\
v_{t}^{s}-u^{s}\left(u_{y}^{s}-v_{x}^{s}\right)+q_{y}^{s}-C\left(v_{x x}^{s}+v_{y y}^{s}\right)=U_{0}(y)\left(A \mathrm{e}^{\mathrm{i} k x}-B \mathrm{e}^{-i k x}\right)\left(\bar{U}_{y}^{s}-\bar{V}_{x}^{3}\right)+\text { c.c. },
\end{gathered}
$$

where the overbar and c.c. stand for the complex conjugate and, in order that all terms in (3.14) (3.15) be of the same order, we are assuming that $\left|u^{s}\right| \sim\left|v^{s}\right| \sim C \sim|A|^{2} \sim|B|^{2}$ and that the slow time scalc for the cvolution of $u^{s}$ and $v^{s}$ is $t \sim|A|^{-2}$; this assumption will be relaxed below. Note that the oscillatory part of the velocity field at order $|A|^{2}$ derives from a potential (see equation $(\Lambda 3)$ in the $\Lambda$ ppendix) and thus does not provide any contribution to the convective terms in (3.14)-(3.15); also, $q_{x}^{s}=q_{y}^{s}=0$ at order $|A|^{2}$ (see equations (A 4) (A 5) in the Appendix) and thus $\left|q_{x}^{s}\right| \sim\left|q_{\gamma}^{s}\right| \sim|A|^{4}$. $U^{3}$ and $V^{3}$ are the velocity components of the oscillatory resonant part (i.e. that part depending on the short time scale as $\exp (\mathrm{i} i \omega t))$ at order $|A|\left(\left|u^{s}\right|+\left|v^{s}\right|\right)$, which satisfy (add to equations (A 9) (A 10) in the Appendix those oscillatory terms proportional to $\mathrm{e}^{\mathrm{i}\left(\mathrm{t}_{\mathrm{r} t} t-i k x\right)}$

$$
\begin{aligned}
& \mathrm{i} \omega U^{3}+V_{0}(y)\left(A \mathrm{c}^{\mathrm{i} k x}+B \mathrm{c}^{\mathrm{i} k x}\right)\left(u_{y}^{s}-v_{x}^{s}\right)=\phi_{x}, \\
& \mathrm{i} \omega V^{3}-U_{0}(y)\left(A \mathrm{c}^{\mathrm{i} k x}-B \mathrm{c}^{\mathrm{i} k x}\right)\left(u_{y}^{s}-v_{x}^{s}\right)=\phi_{y},
\end{aligned}
$$

where we are including on the right-hand sides those terms that derive from a potential (which will not play any role below). Substitution of (3.16) (3.17) into (3.14) and (3.15) and invoking (3.6) yiclds, after some algebra,

$$
\begin{gathered}
u_{t}^{s}+v^{s}\left(u_{y}^{s}-v_{x}^{s}\right)=-\tilde{q}_{x}^{s}+C\left(u_{x \mathrm{x}}^{s}+u_{y y}^{s}\right), \\
v_{t}^{s}-\left[u^{s}+\left(|B|^{2}-|A|^{2}\right) g(y)\right]\left(u_{y}^{s}-v_{x}^{s}\right)=-\tilde{q}_{y}^{s}+C\left(v_{x x}^{s}+v_{y y}^{s}\right),
\end{gathered}
$$

where the function $g\left(=\mathrm{i} \omega_{0}^{-1} \mathrm{~d}\left(U_{0} \bar{V}_{0}\right) / \mathrm{d} y+\right.$ c.c. $)$ coincides with that appcaring in the amplitude equations (3.7) (3.8) (and given by (3.12)), and

$$
\tilde{q}^{s}=q^{s}+\left[i \omega_{0}^{-1} U_{0} V_{0}\left(|A|^{2}-|B|^{2}\right)\left(u_{y}^{s}-v_{x}^{s}\right)+\text { c.c. }\right] .
$$

The momentum equations (3.18) and (3.19) coincide with the usual Navier Stokes equations, except for the vertical body force

$$
\left(0,-\left(|B|^{2}-|A|^{2}\right) g(y)\left(u_{y}^{s}-v_{x}^{s}\right)\right),
$$

which is written in three dimensions as $\boldsymbol{v}^{S d} \times \boldsymbol{\Omega}^{s}$, where $\boldsymbol{\Omega}^{s}$ is the time-averaged vorticity and

$$
v^{S d}=\left(\left(|B|^{2}-|A|^{2}\right) g(y), 0\right) .
$$

is the Stokes drift. The body force (3.20) is sometimes called vortex force generated by $v^{s d}$, and plays an important role on the stability of Langmuir circulations in the upper layers of lakes and ocean (Leibovich \& Paolucei 1981). The Stokes drift appears when 
calculating the Lagrangian (or mass-transport) velocity, $v^{m t}=\left(u^{s}, v^{s}\right)+v^{s d}$, which is the one associated with the time-averaged trajectories of material clements, in contrast with the Fulerian velocity $\boldsymbol{u}^{5}$, which is the time-averaged velocity.

The continuity and momentum equations (3.13), (3.18) and (3.19) apply in the bulk, outside the above-mentioned boundary layers. The boundary conditions at $y=-1$ and 0 are obtained from matching conditions with the slowly varying part of the velocity in the boundary layers, which must be analysed separately. In fact, in the present two-dimensional setting, these boundary conditions are given by the following formulae, first obtained by Schlichting (1932) and Longuet-Higgins (1953),

$$
\begin{gathered}
u^{s}=\alpha_{7}\left[\mathrm{i} A \bar{B} \mathrm{e}^{2 i k x}+c . c .+|B|^{2}-|A|^{2}\right], \quad v^{s}=0 \quad \text { at } \quad y=-1, \\
\partial u^{s} / \partial y=\alpha_{8}\left(|B|^{2}-|A|^{2}\right), \quad v^{s}=0 \quad \text { at } \quad y=0,
\end{gathered}
$$

where we are neglecting terms of the order of $C^{1 / 2}\left(|A|^{2}+|B|^{2}\right)+\left(|A|^{2}+|B|^{2}\right)^{2}$ and the constants $\alpha_{7}$ and $\alpha_{8}$ are

$$
\alpha_{7}=3 \omega_{0} k / \sinh ^{2} k, \quad \alpha_{8}=8 \omega_{0} k^{2} / \tanh k .
$$

Finally, the boundary conditions (2.7) yield

$$
u^{s}, y^{s} \text { and } \tilde{q}^{s} \text { are periodic, of period } L \text {, in } x \text {. }
$$

Equations (3.13), (3.18)-(3.19), (3.22)-(3.23) and (3.25) will be used in $\$ 4$ to analyse the streaming flow. Note that our assumption above on the orders of magnitude of $u^{5}$, $v^{s}, C$ and the slow time variable is not necessary. 'lhis is so because cquations (3.18) and (3.19) have been obtained as a balance between the leading-order effects of inertia (including convection), pressure gradient and viscous dissipation, which are the only ingredicnts on the original momentum equations (2.2)-(2.3) that can contribute to the streaming flow. In particular, the equations remain valid as $C \ll|A|^{2}+|B|^{2}$, which will be a relevant limit below. In fact, viscous eflects cannot be ignored in the analysis of the streaming flow, however large the effective Reynolds number associated with that flow can be. This is so because the streaming flow is forced by a tangential velocity and a tangential stress at the lower and upper boundaries (sec (3.22) and (3.23)), and these two shear mechanisms would not force any flow in the absence of viscosity. Note also that the forcing tangential velocity and stress are independent of viscosity (i.e. of $C$ ) to leading order, and thus they converge to non-zero values as $C \rightarrow 0$. Thus, this is a good example to illustrate that the limits $C \rightarrow 0$ and $|A|+|B| \rightarrow 0$ do not commute, as anticipated in $\$ 2.1$.

\section{Coupled spatial phase-streaming flow equations}

The coupled evolution of the surface waves and the streaming flow is given by the system of equations and boundary conditions (3.7) (3.8), (3.13), (3.18) (3.19), (3.22), (3.23) and (3.25). These equations are first reduced in $\$ 4.1$ to two systems of cquations that apply after a transient in the slow time scale associated with viscous dissipation. The first system is decoupled and yields the evolution of the surface waves to a drifting SW, which is determined up to a spatial phase. Once the amplitude of the surface wave is determined, the second system gives the coupled evolution of the spatial phase and the streaming llow. 

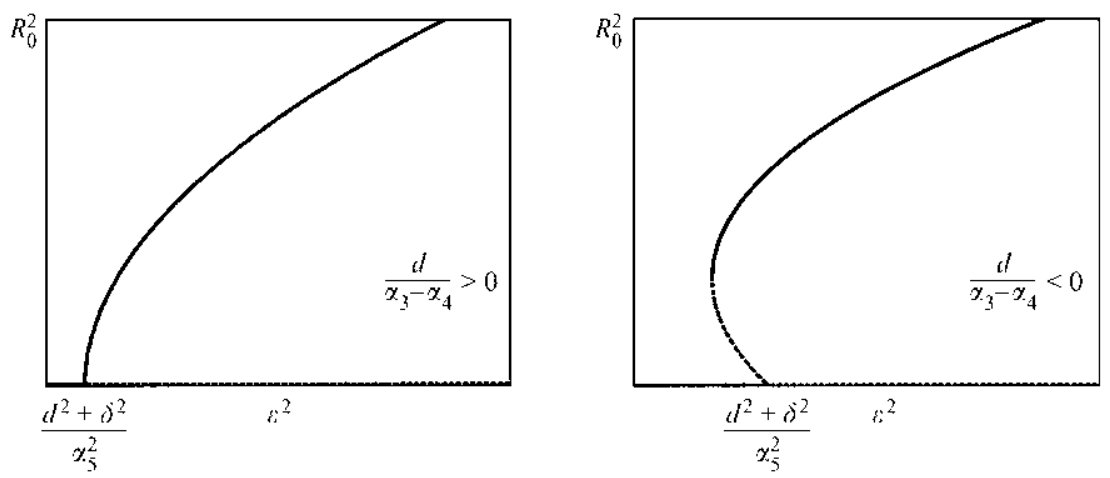

FIGURE 4. Amplitude of - , the stable and ---, unstable shifted SWs (non-trivial steady states of (4.3)-(4.4)) in terms of the forcing amplitude.

4.1. Derivation of the coupled spatial phase-streaming flow equations

The effect of the streaming flow on the complex amplitudes can be decoupled using the new complex amplitudes and the spatial phase, defined as

$$
A=A_{0} \mathrm{e}^{-i k \psi}, \quad B=B_{0} \mathrm{e}^{i k \psi},
$$

with

$$
\mathrm{d} \psi / \mathrm{d} t=\left[\alpha_{6} /(k L)\right] \int_{-1}^{0} \int_{0}^{L} g(y) u^{s}(x, y, t) \mathrm{d} x \mathrm{~d} y .
$$

Substituting these into (3.7)-(3.8), $A_{0}$ and $B_{0}$ are seen to satisfy

$$
\begin{aligned}
& A_{0}^{\prime}=\left[-\delta-\mathrm{i} d+\mathrm{i} \alpha_{3}\left|A_{0}\right|^{2}-\mathrm{i} \alpha_{4}\left|B_{0}\right|^{2}\right] A_{0}+\mathrm{i} \varepsilon \alpha_{5} \bar{B}_{0}, \\
& B_{0}^{\prime}=\left[-\delta-\mathrm{i} d+\mathrm{i} \alpha_{3}\left|B_{0}\right|^{2}-\mathrm{i} \alpha_{4}\left|A_{0}\right|^{2}\right] B_{0}+\mathrm{i} \varepsilon \alpha_{5} \bar{A}_{0} .
\end{aligned}
$$

These are the standard amplitude equations that have been systematically used in the weakly nonlinear description of spatially uniform Faraday waves. Their solutions relax to steady states. This property is always taken for granted in the literature; the proof is somewhat standard, but non-trivial and outside the scope of this paper. The zero steady state of (4.3) (4.4) is unstable whenever the forcing amplitude $\varepsilon$ is larger than its threshold value, i.e. $\varepsilon>\varepsilon_{c} \equiv \sqrt{\delta^{2}+d^{2}} /\left|\alpha_{5}\right|$. If $\alpha_{3}-\alpha_{4} \neq 0$, as we assume hereinafter (otherwise some higher-order terms must be added to (4.3)-(4.4)), the remaining steady states are of the form

$$
A_{0}=B_{0}=R_{0} \mathrm{e}^{\mathrm{i} \phi_{0},},
$$

where $\phi_{0}$ is an arbitrary temporal phase and $R_{0}>0$ is given by

$$
R_{0}^{2}=\left[d \pm\left(\alpha_{5}^{2} z^{2}-\delta^{2}\right)^{1 / 2}\right] /\left(\alpha_{3}-\alpha_{4}\right) .
$$

These steady states build a branch that bifurcates from the flat state at $\varepsilon=\varepsilon_{c}$ and is either monotone (if $d /\left(\alpha_{3}-\alpha_{4}\right)<0$ ) or C-shaped, see figure 4 ; in the latter case, the intermediate solutions are unstable. Note that since (4.5) holds, the surface wave associated with these steady states is a drifting SW with an amplitude $R_{0}$; and $\psi$ can be seen as its spatial phase, for to leading order the free surface deflection is given by

$$
f(x, t)=4 R_{0} \cos \left(\omega t+\phi_{0}\right) \cos [k(x-\psi)],
$$

as readily obtained from (3.4), (4.1) and (4.5). The drift velocity of the waves $d \psi / d t$ is due to the streaming flow (4.2) and it is essentially different from that analysed in 
Douady et al. (1989) that was due to the breaking of the spatial reflection symmetry of the waves, namely it required $|A| \neq|B|$.

Thus, after a transient, we can assume that (4.5) holds and rewrite (3.13), (3.18)(3.19), (3.22) (3.23), (3.25) and (4.2) as

$$
\begin{gathered}
\tilde{u}_{x}+\tilde{v}_{y}=0, \\
\partial \tilde{u} / \partial \tau+\tilde{v}\left(\tilde{u}_{y}-\tilde{v}_{x}\right)=-\tilde{q}_{x}+\operatorname{Re}^{-1}\left(\tilde{u}_{x x}+\tilde{u}_{y y}\right), \\
\partial \tilde{v} / \partial \tau-\tilde{u}\left(\tilde{u}_{y}-\tilde{v}_{x}\right)=-\tilde{q}_{y}+\operatorname{Re}^{-1}\left(\tilde{v}_{x x}+\tilde{v}_{y y}\right), \\
\tilde{u}=-\sin [2 k(x-y)], \quad \tilde{v}=0 \quad \text { at } \quad y=-1, \\
\partial \tilde{u} / \partial y=\tilde{v}=0 \quad \text { at } \quad y=0,
\end{gathered}
$$

$\tilde{u}, \tilde{v}$ and $\tilde{q}$ are $x$-periodic, of period $L=2 m \pi / k$,

$$
\mathrm{d} \psi / \mathrm{d} \tau=L^{\perp} \int_{1}^{0} \int_{0}^{L_{L}} G(y) \tilde{u}(x, y, \tau) \mathrm{d} x \mathrm{~d} y,
$$

in terms of the rescaled variables

$$
\tau=\operatorname{ReCt}, \quad \tilde{u}=u^{s} /(\operatorname{ReC}), \quad \tilde{v}=v^{s} /(\operatorname{ReC}), \quad \tilde{q}=\tilde{q}^{s} /(\operatorname{ReC})^{2},
$$

where $m \geqslant 1$ is an integer and the function $G$ and the Reynolds number Re are

$$
G(y)=2 k \cosh [2 k(y+1)] / \sinh 2 k, \quad R e=6 R_{0}^{2} \omega_{0} k /\left(C \sinh ^{2} k\right) .
$$

Note that $L{ }^{1} \iint G \mathrm{~d} x \mathrm{~d} y=1$, which is consistent with the invariance of (4.8)-(4.10) and (4.14) under the action $x \rightarrow x+c \tau, \tilde{u} \rightarrow \tilde{u}+c, y=\psi+c \tau$, as required by the invariance of (2.1) (2.3) under Galilean transformations.

Equations (4.8)-(4.14) will be called coupled spatial phase-streaming flow (CSPSF) equations, and $\tilde{u}_{0}=\mathrm{d} \psi / \mathrm{d} \tau$ can be seen as a drift velocity of the drifting SWs. Those solutions of the CSPSF equations with $\tilde{u}_{0}=$ constant are precisely uniformly drifted SWs, as are those encountered by Douady et al. (1989).

The CSI'SF equations (4.8) (4.14) depend only on the wavenumber $k$, spatial period $L=2 m \pi / k$ and the cffective Reynolds number Re. The latter is proportional to the square of the wave steepness, $R_{0} k$, which must be small. Since $C$ is also small (of the order of $10^{4}$ for water in containers of depth of the order of $1 \mathrm{~cm}$, as in the experiment by Douady et al. 1989), $R e$ can vary in a wide range. Assuming that $T$ is not large (which is true for gravity waves), $\left(R_{0} k\right)^{2} \leqslant 0.1$ and $C \geqslant 10^{6}$, and using (3.2) and (4.16)

$$
0 \leqslant R e \leqslant 12 \times 10^{4} /\left(\omega_{0} \sinh 2 k\right) .
$$

4.2. Large time dynamics of the coupled spatial phase-streaming flow equations T'he CSPSF equations (4.8)-(4.14) are invariant under the symmetrics

$$
\begin{gathered}
x \rightarrow x+c, \quad y \rightarrow w+c, \\
x \rightarrow-x, \quad \tilde{u} \rightarrow-\tilde{u}, \quad w \rightarrow-\psi, \\
x \rightarrow x+\frac{1}{2} L .
\end{gathered}
$$

The first two symmetries come from the invariance of the original problem (2.1) (2.7) under horizontal translation and reflection. The reflection-symmetric attractors (invariant under (4.19) after a translation, referred to as r-symmetric, will be called locally or globally r-symmetric depending on whether they are r-symmetric for all $\tau$ 

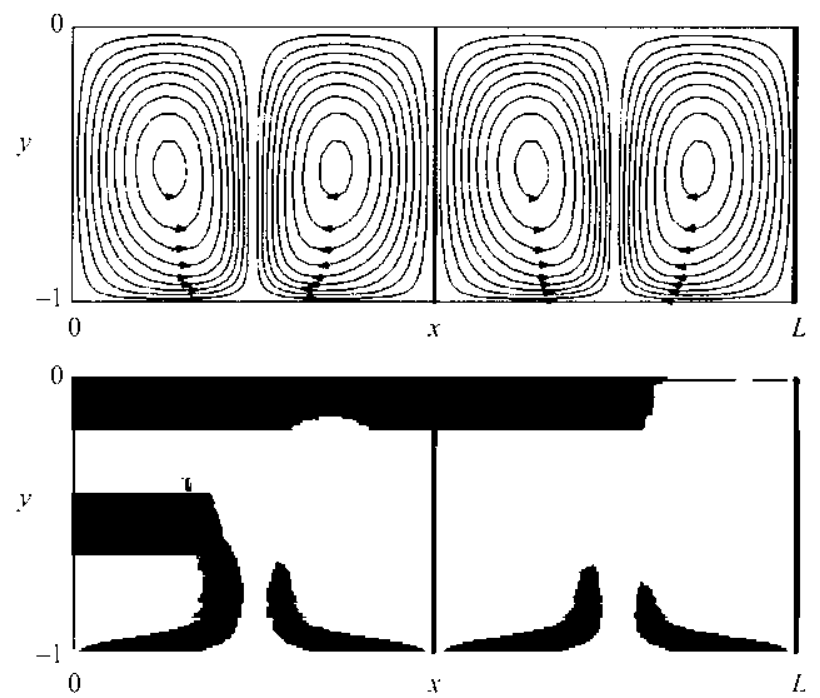

FIGURE 5. Streamlines and vorticity colour (red = positive vorticity, blue = negative vorticity) map of the basic steady state of (4.8) (4.14) for $R e=260, k=2.37$ and $m=1$ ( $L=2.65$ ). Thick vertical lines are as in figure 2.

or they exhibit an r-symmetric orbit in phase space. The last symmetry (4.20) will be useful when considering the dynamics of (4.8)-(4.14) for varying values of $L$. In particular (when doubling $L$ ), attractors that are invariant under (4.20) are also present in a cell of width $\frac{1}{2} L$, and will be called $\left(\frac{1}{2} L\right)$-periodic.

The analysis of the attractors of the CSPSF equations (4.8)-(4.14) must rely on numerics. The equations have been discretized by means of a spectral method in the horizontal coordinate, an equispaced second-order finite-difference scheme in the vertical coordinate, and a second-order semi-implicit method to march in time (Canuto et al. 1988). The cheapest calculations, for $R e<450$ and $m=1$, require 128 Fourier modes and spatial and temporal step sizes $\Delta y=\Delta \tau=0.01$. In order to avoid too expensive computations we shall restrict ourselves to the ranges $1 \leqslant m \leqslant 10$ and $0<R e<900$, although $R e$ can take much larger values within the scope of the theory, especially for moderate values of $k$, see (4.17).

For small $R e$, the CSPSF equations become linear and exhibit a unique attractor, which is an r-symmetric steady state; thus it exhibits no drift, namely $\psi=$ constant, according to (4.14), and corresponds to a standard SW. The streamlines are as those plotted in figures $2(a)$ and 5 for $m=1$ and two representative values of $(k, R e)$; for comparison with other attractors, the associated vorticity $\left(\tilde{\Omega}=\tilde{v}_{x}-\tilde{u}_{v}\right)$ colour map is also given in figure 5. As $R e$ is increased, this steady state becomes unstable through a Hopf bifurcation at a threshold value $R e=R e^{\prime}$, which is plotted $v s . k$ with a solid line in figure 6 . This plot has been obtained for $m=1$ but has been checked to remain unchanged for $m=10$. This instability threshold is always larger than that obtained when the effect of the spatial phase is ignored (dashed line); thus, the coupling to the spatial phase has a stabilizing effect on the streaming flow. Note that the instability occurs when $R e$ is large. Thus, we can distinguish a convective timescale, $\tau \sim 1$ and a viscous timescale, $\tau \sim R e$. The frequency of the unstable modes at threshold is of order unity, showing that this instability is associated mainly with convective effects.

Now we consider two representative bifurcation diagrams using $R e$ as a bifurcation parameter, which is varied in small steps. At each step we take as the initial condition 


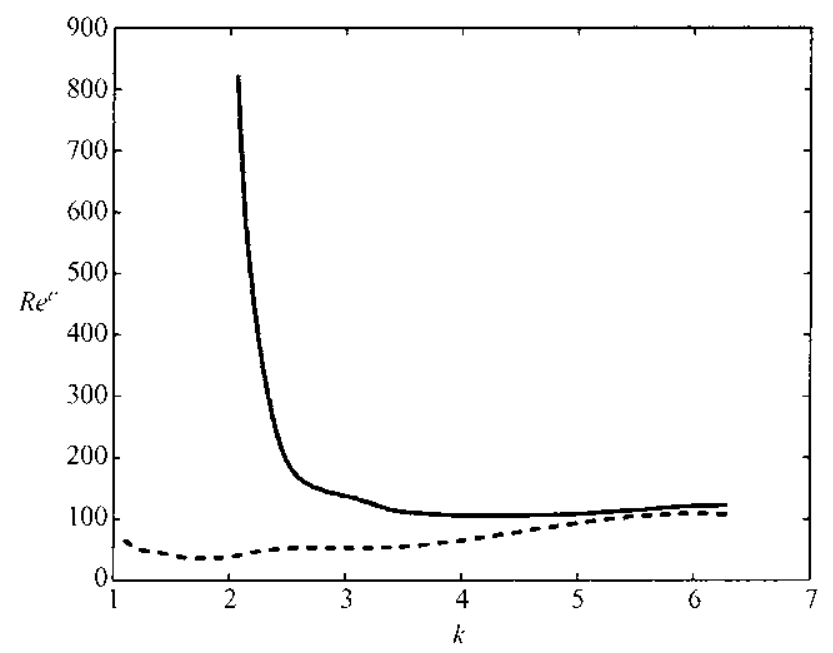

Figure 6. Stability diagram of the basic solution. - Hopf bifurcation of the CSPSF equations (4.8)-(4.14) and --- , pitchfork bifurcation for the uncoupled equations (4.8)-(4.13), with $y=0$.

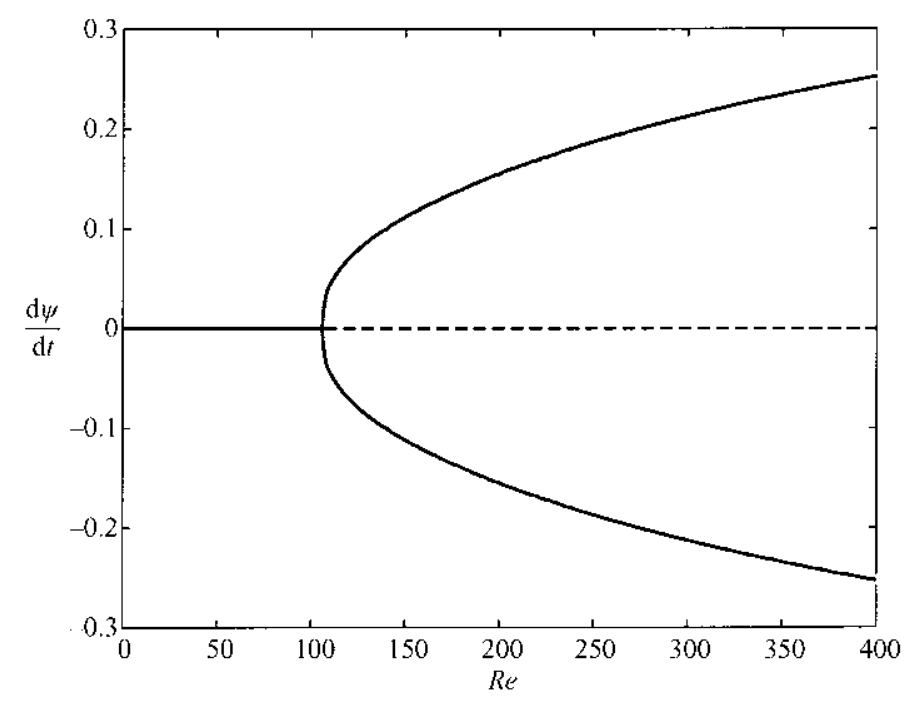

FIGURE 7. Bifurcation diagram of the CSPSF equations for $k=4$ and $m=1\left(L=\frac{1}{2} \pi\right)$.

a point in the attractor for the previous step plus a small perturbation containing all Fourier modes. We integrate the CSPSF equations in an initial time interval to climinate transient behaviours and then plot the following quantities, which are useful to appreciate r-symmetric and $\left(\frac{1}{2} L\right)$-periodic attractors

$$
\left\|y^{\prime}\right\|_{r}=\psi^{\prime}\left(\tau_{j}\right), \quad\|\Omega\|_{p}=\left\|\Omega^{\text {odd }}\left(\tau_{j}\right)\right\|_{L_{2}},
$$

where $\tau_{j}$ are the stationary points of $\psi^{\prime}$ (namely $\psi^{\prime \prime}\left(\tau_{j}\right)=0$ ), $\mid \cdot \|_{L_{2}}$ is the $L_{2}$ norm and

$$
\Omega^{\text {odd }}=\sum_{n=\text { odd }} \Omega_{j} \mathrm{e}^{\mathrm{i} 2 \pi n \times / L} \quad \text { if } \quad \Omega=\sum_{n=-x}^{n=x} \Omega_{j} \mathrm{e}^{\mathrm{i} 2 \pi n \cdot / L}
$$



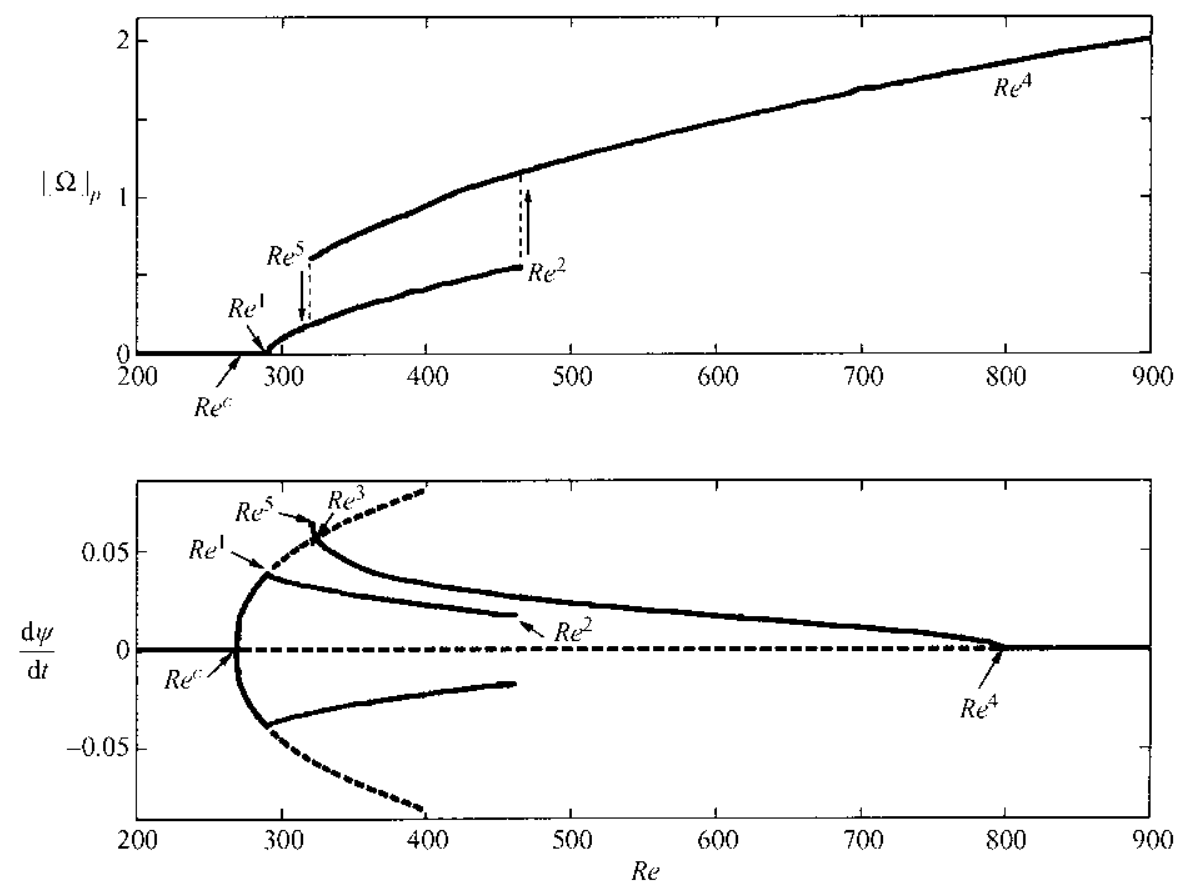

Figure 8. Bifurcation diagram of the CSPSF equations for $k=2.37$ and $m=1(L=2.65)$.

The bifurcation diagram for $k=4$ and $m=1$ is plotted in figure 7 , where according to the restriction (4.17) only the interval $0<R e<160$ is of interest here. The IIopf bifurcation at $R e=R e^{c} \simeq 105$ is supercritical and the resulting limit cycle is (not locally r-symmetric but is) globally r-symmetric (the plot of $\left|y^{\prime}\right|$, is symmetric) and locally $\left(\frac{1}{2} L\right)$-periodic $\left(\|\Omega\|_{p} \equiv 0\right)$. The associated pattern is an array of laterally oscillating eddies, whose size also oscillates, and is qualitatively similar to that in figure $9(a)$. The period varies between 46 and 79 as $R e$ increases from 105 to 160 . Finally, we have checked that this bifurcation diagram remains unchanged as $m$ is increased up to $m=10$.

As an example of a more complex bifurcation diagram, we give in figure 8 that obtained for $k=2.37$ and $m=1$ (thus $L=2.65$ ). The Hopf bifurcation at $R e=R e^{c} \simeq 270$ is again supercritical. The resulting limit cycle is $\left(\frac{1}{2} L\right)$-periodic and globally r-symmetric, like that in figure $9(a)$, where for illustration a thick vertical line is plotted at the nodes of the primary surface wave, which are

$$
x_{\text {node }}=\psi+\pi /(2 k) \text { and } x_{\text {nodc }}=\psi+3 \pi /(2 k) \text {. }
$$

according to (4.7); note that the nodes oscillate only slightly. This branch of limit cycles losses stability at $R e=R e^{1} \simeq 291.5$ (the unstable part, plotted with dashed lines, has been computed imposing $\left(\frac{1}{2} L\right)$-periodicity), where a new family of limit cycles bifurcates that are still globally $r$-symmetric but no longer $\left(\frac{1}{2} L\right)$-periodic $\left(\|\left.\Omega\right|_{p}\right.$ is no longer zero). One of these is given in figure $9(b)$, where the lack of $\left(\frac{1}{2} L\right)$-periodicity is apparent and it is seen that this limit cycle again involves only a slight motion of the surface wave. Note that this is a supercritical symmetry breaking bifurcation that produces the solution in figure $9(b)$ and also that obtained upon applying a $\left(\frac{1}{2} L\right)$-translation, but both solutions yicld the same point in figure 8 . Note also that the plot of $\|\left.\Omega\right|_{\text {. }}$ shows only one point for each limit cycle; this is so because the 

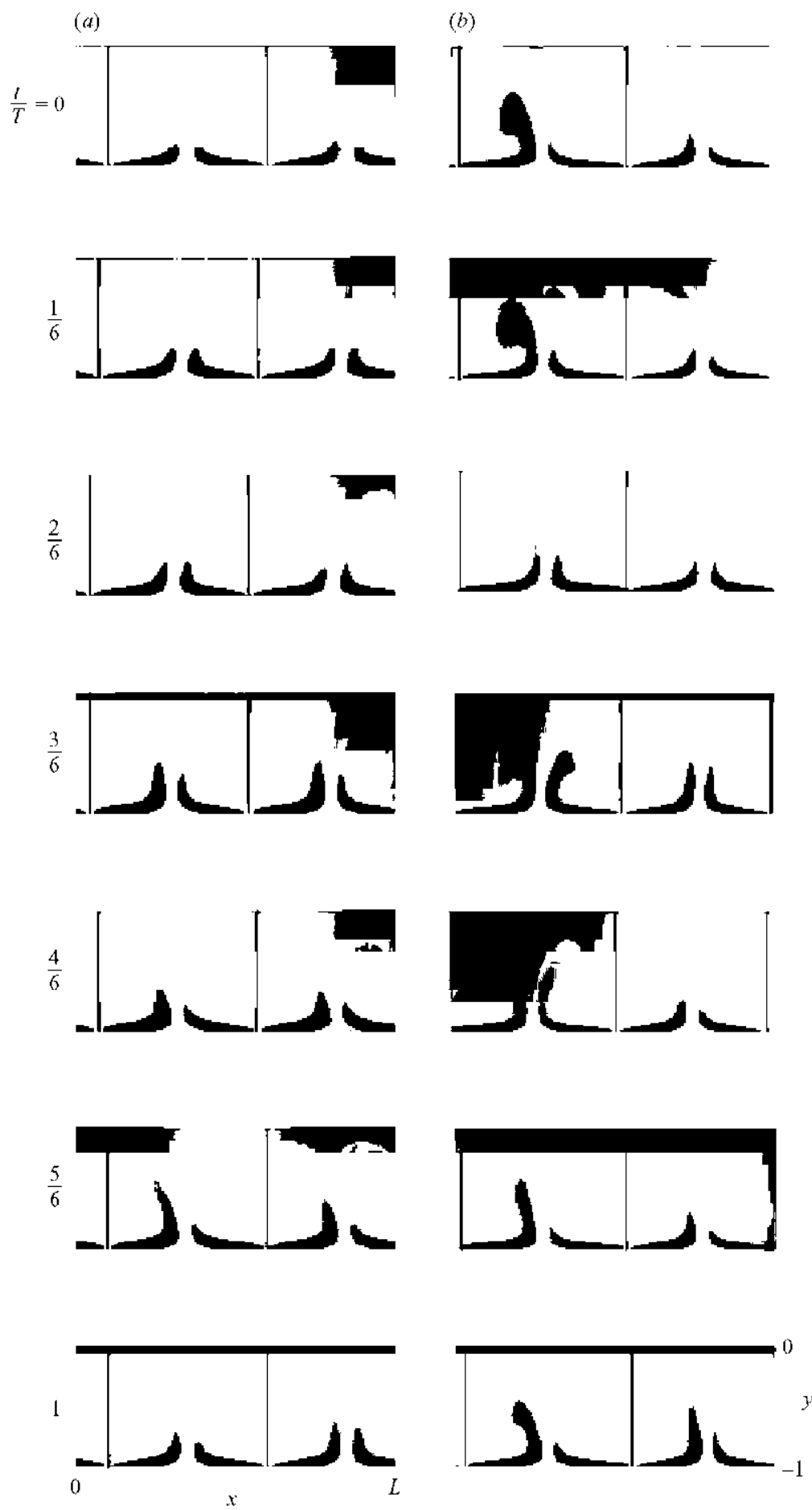

Figure 9. Vorticity colour maps at eciuispaced values of $\tau$ in the stable limit cycle for $k=2.37$, $m=1$ and $(a) R e=290$ and $(b) R e=400$. Thick vertical lines are as in figure 2 . 

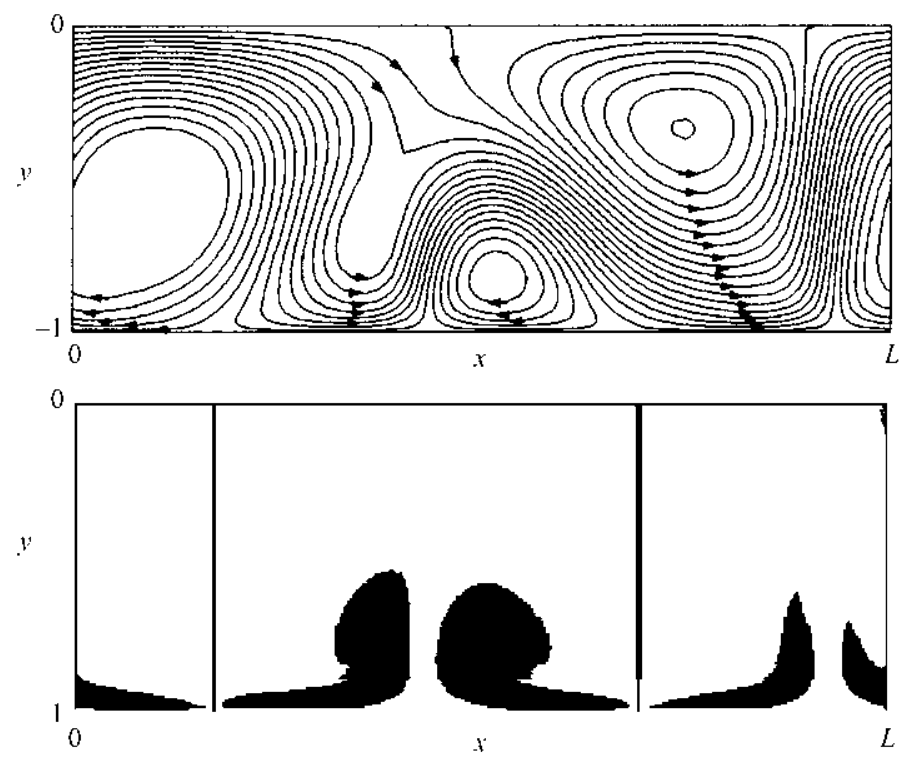

FIGURE 10. Streamlines and vorticity colour map in moving axes of the streaming flow produced by the drifted standing wave at $R e=325$. Thick vertical lines are as in figure 2 .
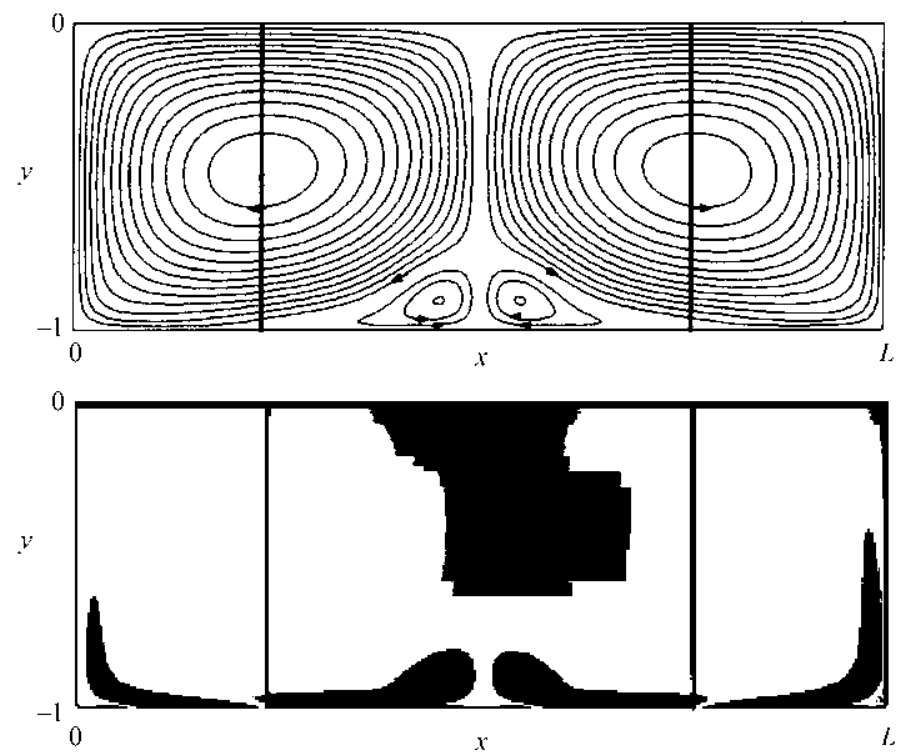

Figure 11. Streamlines and vorticity colour map of the stable steady state in figure 8 at $R e=850$. Thick vertical lines are as in figure 2 .

velocity field at two values of $\tau$ where $\psi^{\prime}$ reaches a maximum and a minimum are obtained from each other by a translation and an $x$-reflection. This non $\left(\frac{1}{2} L\right)$-periodic limit cycle is stable in the interval $R e^{1}<R e<R e^{2} \simeq 466$, where it loses stability and the system jumps to a new branch of travelling wave solutions, which exhibit a constant drift velocity, $\psi^{\prime}=\tilde{u}_{0}$, and are steady in a reference frame moving at a speed $\tilde{u}_{0} ;$ the streamlines and vorticity contours in the moving frame (using the new 

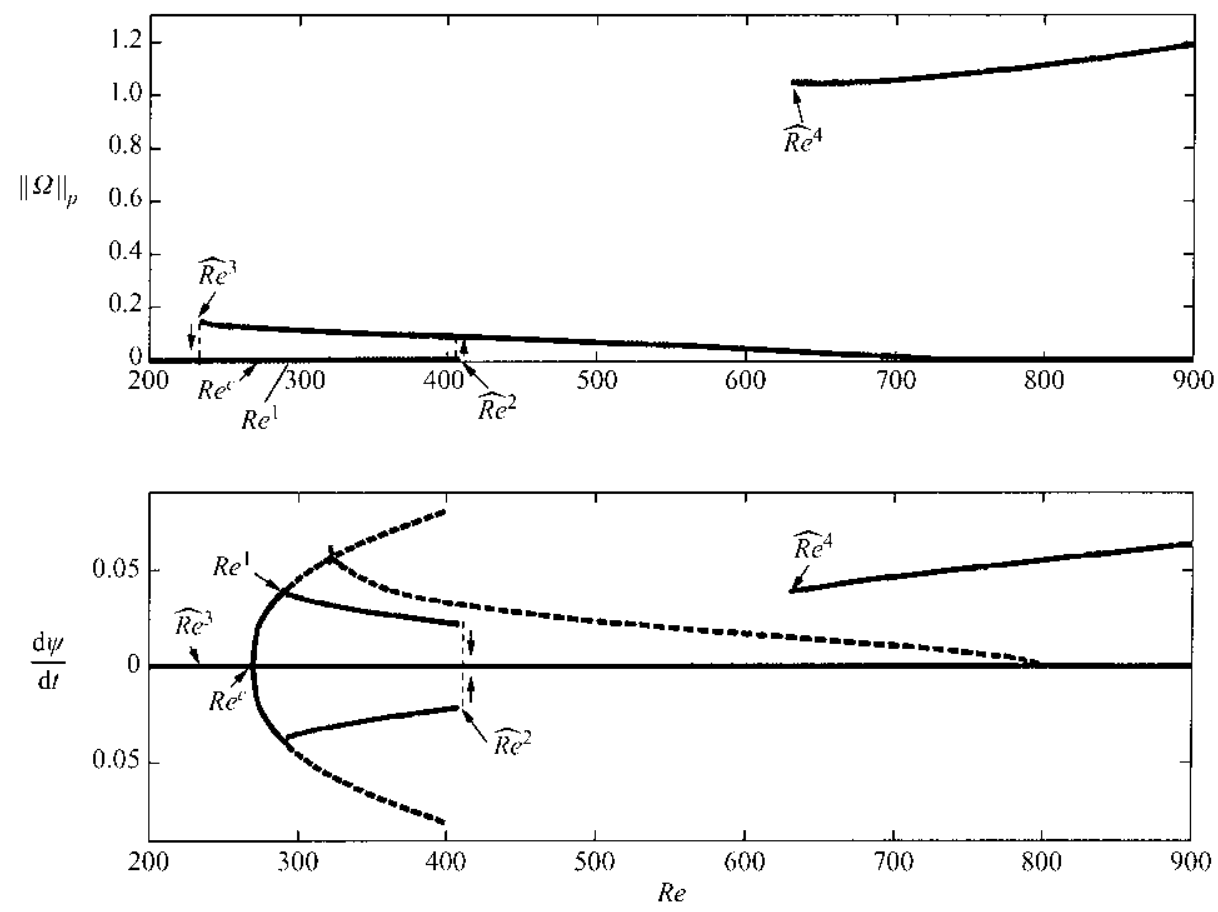

FIGURE 12. Bifurcation diagram of the CSPSF equations for $k=2.37$ and $m=2$.

horizontal velocity $\tilde{u}-\tilde{u}_{0}$ ) are plotted in figure 10 . Thus, these attractors correspond to the drifted SWs mentioned above, and are neither $r$-symmetric (which could not be because reflection symmetry is lost in the moving frame) nor $\left(\frac{1}{2} L\right)$-periodic. In fact, this new branch of solutions exists in a wide range $323 \simeq R_{c}^{3}<R e<R e^{4} \simeq 800$ and thus the system exhibits hysteresis. Note that the drift velocity $\tilde{u}_{0}=w^{\prime}$ decreases as $R e$ increases. At $R e=R e^{3}$ the drifted SWs lose stability at a supercritical Hopf bifurcation to limit cycles that are neither r-symmetric nor $\left(\frac{1}{2} L\right)$-periodic and in turn lose stability at $R e=R e^{5} \simeq 319$, where the system jumps to the branch of $\mathrm{r}$-symmetric but not $\left(\frac{1}{2} L\right)$-periodic limit cycles described above. At $R e=R e^{4}$ instead, the drifted SWs disappear at a supercritical (reversed) bifurcation that yields new r-symmetric (but not $\left(\frac{1}{2} L\right)$-periodic) steady states that are like that in figure 11 . Thus, these are quite different from the $\left(\frac{1}{2} L\right)$-periodic steady state that existed for $R e<\operatorname{Re}^{c}$ (cf. figure 4). Note that when the bifurcation at $R e=R e^{4}$ is seen for decreased values of $R e$, it is a standard parity-breaking bifurcation (Greene \& Kim 1988; Dangelmayr, Hettel \& Knobloch 1997), which appears in systems invariant under reflection and translation and produces a drift (like the one here) of the patterns under generic conditions.

If $k=2.37$, as in figure 8 , but $m=2$ then we obtain a somewhat different bifurcation diagram plotted in figure 12. The bifurcations at $R e=R e^{r}$ and $R e=R e^{1}$ remain unchanged, but now the branch of non $\left(\frac{1}{2} L\right)$-periodic limit cycles born at $R e=R e^{1}$ loses stability at $R e=\widehat{R e}^{2} \simeq 410$. This bifurcation was not present in the case $m=1$ (cf. figure 8), where the instability happened at $R e=R e^{2} \simeq 466<\widehat{R e}^{2}$ and the system jumped to a branch of travelling waves that are now unstable and plotted with a dashed line in figure 12. At $R e=\widehat{R e}^{2}$, the system jumps to a steady state whose 

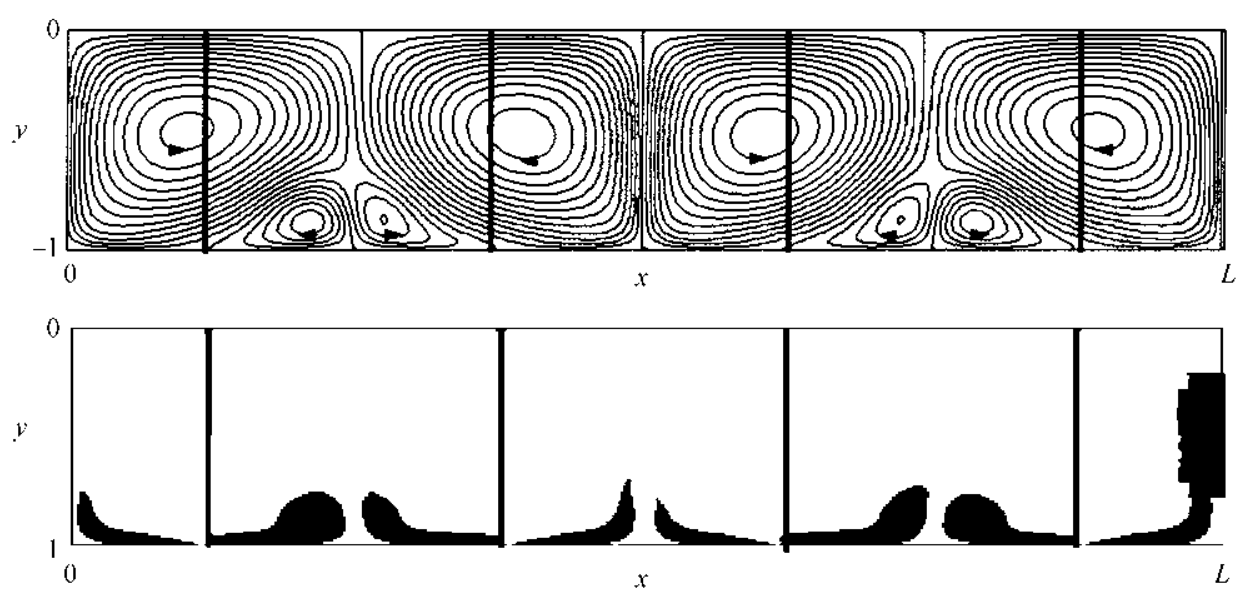

Figure 13. Streamlines and vorticity colour map of the stable steady state in figure 12 at $R e=418$. Thick vertical lines are as in figure 2 .
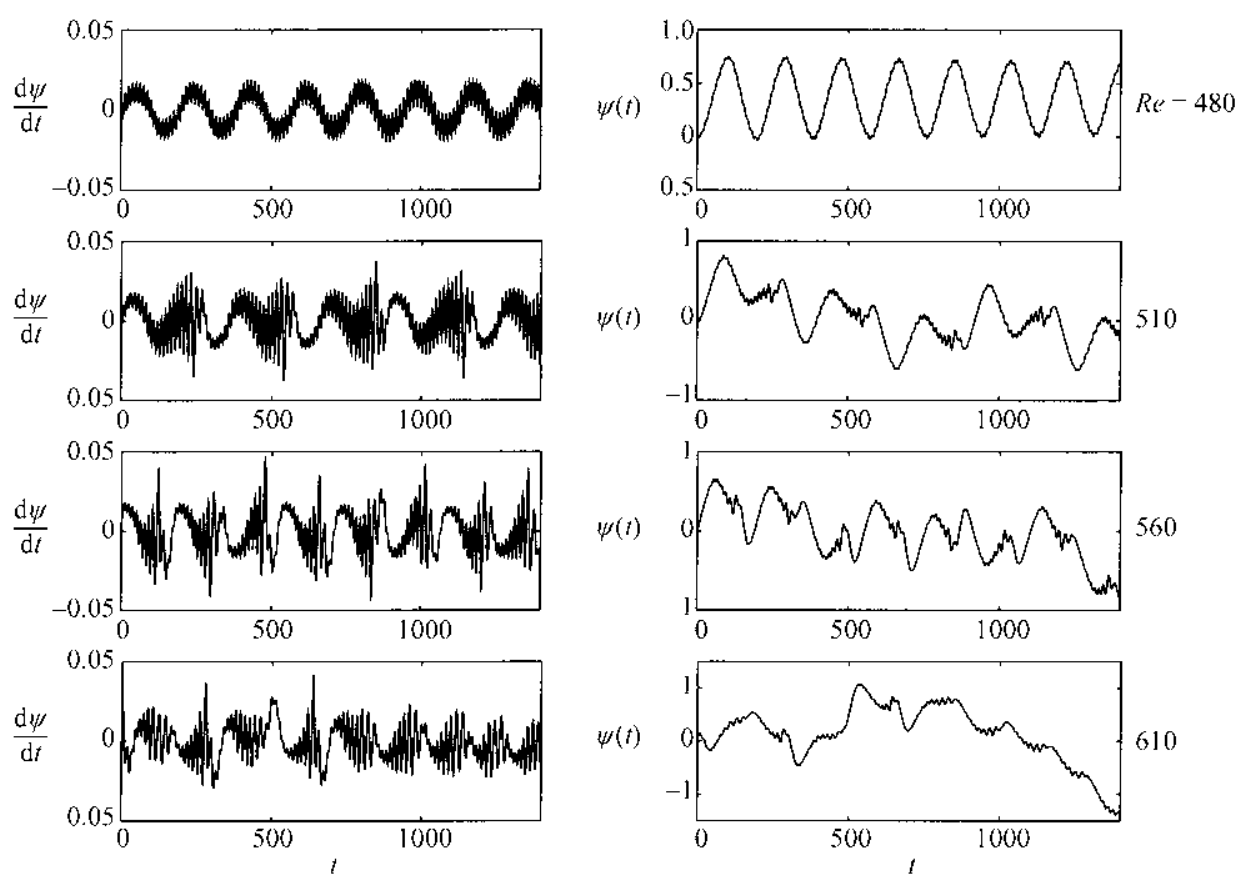

Figure 14. Plots $\psi^{\prime}$ us. $\tau$ and $\psi$ es. $\tau$ for some representative more complex attractors for $k=2.37$, $m=2$ and the indicated values of $R e$.

streamlines are plotted in figure 13. This steady state is locally r-symmetric (thus exhibits no drift) but not $\left(\frac{1}{2} L\right.$ )-periodic; thus it was not present for $m=1$ and differs from the steady states found there, even though the streamlines are quite similar to those in figure 11. These new steady states are stable only for $\operatorname{Re}>\widehat{\operatorname{Re}}^{3} \simeq 232$, where the basic steady state is recovered. Thus, the system exhibits considerable hysteresis. The attractors described so far are the only ones that are obtained when slowly moving $R e$ up and down; but, in addition, if a non-small perturbation is added to the steady state at large $R e$ we obtain the new branch of drifted SWs plotted in 
figure 12. These are not $\left(\frac{1}{2} L\right)$-periodic and thus are not present at $m=1$. Furthermore their drift velocity increases as Re increases, in contrast to the drifted SWs in figure 8. The drifted SWs lose stability as $R e$ is decreased, at $R e=\widehat{R e}^{4} \simeq 620$. For smaller $R e$, in the range $475<R e<\widehat{R e}^{4}$, the system shows more complex (periodic, quasiperiodic, chaotic) attractors, like those plotted in figure 14; note that these usually show oscillations in both the convective $(\tau \sim 1)$ and the diffusive $(\tau \sim R e)$ timescales. IIowever, we neither pursue these nor look for additional attractors. Instead, given the strong differences between the bifurcation diagrams in figures 8 and 12 , the natural question arises of which part of the bifurcation diagrams in these figures remain at large aspect ratio. We have considered the (computationally expensive) case $m=10$ and found that the first two bifurcations at $R e^{c}$ and $R e^{1}$ and the bifurcated branches remain unchanged for $\operatorname{Re}<\widehat{\operatorname{Re}}^{2}$.

\section{Conclusions}

We have derived in $\$ 2$ a system of coupled amplitude-streaming flow equations for the wcakly nonlincar cvolution, at small viscosity, of parametrically excited surface waves in a two-dimensional liquid layer supported by a horizontal plate, which is vertically vibrated. We imposed periodic boundary conditions in the horizontal direction, intending to model an annular container. For simplicity, we considered a monochromatic surface wave and ignored wave modulation. We have derived from first principles the amplitude equations, which are two complex ODEs that include the usual terms associated with inertia, linear damping, detuning, conservative cubic nonlinearity and forcing, and a new term that accounts for coupling with the streaming flow. The streaming flow is described by the usual continuity and NavierStokes equations, except for a horizontal vortex force driven by the Stokes drift. The boundary conditions include a tangential velocity and a tangential stress at the lower and upper boundaries, respectively, which are independent of viscosity and depend quadratically on the complex amplitudes, according to well-known formulae duc to Schlichting (1932) and Longuct-Higgins (1953). These cquations have been further simplified by taking advantage of the fact that the surface wave evolves, in the time scale associated with surface-wave damping, to a drifting SW, which is completely determined (independently of the streaming flow) up to a spatial phase. Thus, the problem is reduced to a set of coupled spatial phase-streaming flow (CSPSF) cquations. These cquations are invariant under horizontal translation and reflection, and depend on an effective Reynolds number $R e$, the wavelength of the surface waves $k$ and the length of the container, $L=2 m \pi / k$ for some integer $m$. The CSISSF equations have been numerically integrated and the range $0<k<6,0<\operatorname{Re}<900$, for $m=1,2$ and 10, has been explored. The main conclusions are:

(i) If the coupling of the streaming flow with the surface waves is removed, then the surface wave nodes are stationary. The associated streaming flow lose reflection symmetry for moderate values of $R e$, and the resulting non-symmetric flow should produce a drift of the waves, which is in contradiction with ignoring the effect of the streaming flow on the surface waves. The coupling to the streaming flow prevents this instability (and stabilizes the SWs) for moderate Re, but these SWs cxhibit a Hopf bifurcation at a larger value of $R e$. This primary bifurcation seems to be present for all $k$ and to remain unchanged at large aspect ratio.

(ii) In the derivation of these equations we have seen that it is inconsistent to ignore a priori the effect of the streaming flow and retain the usual cubic nonlinearity, because both of them are of the same order. Also, the streaming flow provides a 
natural mechanism to produce (or prevent) drift instabilities without the need to consider higher-order (quintic, ctc.) terms (as in, c.g. Crawford, Knobloch \& Riecke 1990; Fauve et al. 1991).

The following conclusions are also relevant:

(iii) For some values of $k$ there are some additional bifurcations to drifted SWs (which are standard SWs in a reference frame moving at a constant speed), nonsymmetric limit cycles and more complex oscillatory attractors, but these depend on both $k$ and $L$.

(iv) In oscillatory attractors, oscillations occur frequently in the convective time scalc, though these are sometimes modulated in the slower viscous time scale. $\Lambda \mathrm{c}$ cording to our non-dimensionalization in $\$ 1$, the latter time scale is of the order of $h^{2} / v$, where $h$ is the height of the container and $v$ is the kinematic viscosity; this is of the order of $1 \mathrm{~min}$ for water if $h=0.8 \mathrm{~cm}$ (as in Donady et al. 1989), but it can be much larger than that for larger $h$ (e.g. of the order of $10^{4} \mathrm{~s}$, that is, of the order of hours if $h=10 \mathrm{~cm}$ ).

As to the relevance of the results above in explaining/predicting experimental bchaviours, the restricted two-dimensional formulation above prevents quantitative predictions, but the main conclusion, (ii), obviously applies in the three-dimensional case as well. The main new ingredient in three-dimensions is the presence of the lateral walls, whose attached boundary layers also produce a horizontal tangential streaming velocity, which enhances the coupling between the surface waves and the streaming nlow. The remaining qualitative conclusions are also expected to apply in threcdimensions. In fact, the drifted standing waves and the various oscillatory solutions obtained above are reminiscent of the steadily rotating and laterally oscillating structures found experimentally by Douady et al. (1989), although no further comparison is possible because no quantitative results on these structures were provided.

Some additional physical ellects neglected above could have a role in enhancing drift instabilities in two-dimensions. For instance, surface contamination (which should be expected in water unless great care is taken in the experimental set-up) changes dramatically the structure of the oscillatory boundary layer attached to the free surface (IIenderson \& Miles 1994; Nicolás \& Vega 2000), which becomes somewhat similar to the Stokes boundary layer attached to solid walls, and also yields a forcing tangential velocity for the streaming flow. Thus, the structure of the streaming flow associated with SWs completely changes and its stability properties might change as well, but this is well ahead the scope of this paper.

Rescarch partially supported by DGI and N $\Lambda$ S $\Lambda$, under Grants BFM2001-2363 and NAG3-2152.

\section{Appendix. Nonlinear terms in the amplitude equations}

Iere, we derive those terms in the amplitude equations (3.7)-(3.8) that account for cubic nonlinearity, parametric forcing and coupling with the streaming flow. As explained in $\$ 2.1$, these terms can be derived in the strictly inviscid limit

$$
C=0 \text {, }
$$

when the boundary layers disappear and the analysis is much simpler. For the sake of clarity, we rescale the complex amplitudes, the forcing amplitude and the slowly varying part of the velocity components as

$$
(A, B)=\mu\left(A_{0}, B_{0}\right), \quad \varepsilon=\mu^{2}, \quad\left(u^{s}, v^{s}, q^{s}, f^{s}\right)=\mu^{2}\left(u_{1}^{s}, v_{1}^{s}, q_{1}^{s}, f_{1}^{s}\right),
$$


where $0 \ll \mu \ll 1$ and $\left|A_{0}\right|,\left|B_{0}\right|,\left|u_{1}^{s}\right|$ and $\left|v_{1}^{s}\right|$ are treated as $O(1)$ quantities. Then the expansions (3.4) are rewritten as

$$
\begin{aligned}
u & =\mu \mathrm{e}^{\mathrm{i} \mu_{0} t} U_{0}\left(A_{0} \mathrm{e}^{i k x}-B_{0} \mathrm{e}^{-i k x}\right)+\text { c.c. }+\mu^{2} u_{1}+\mu^{3} u_{2}+\cdots, \\
(v, q) & =\mu \mathrm{e}^{\mathrm{i} u_{0} t}\left(V_{0}, Q_{0}\right)\left(A_{0} \mathrm{e}^{\mathrm{i} k x}+B_{0} \mathrm{e}^{-i k x}\right)+\text { c.c. }+\mu^{2}\left(v_{1}, q_{1}\right)+\mu^{3}\left(v_{2}, q_{2}\right)+\cdots, \\
f & =\mu \mathrm{e}^{\mathrm{i} \omega_{0} t}\left(A_{0} \mathrm{e}^{i k x}+B_{0} \mathrm{e}^{-i k x}\right)+\text { c.c. }+\mu^{2} f_{1}+\mu^{3} f_{2}+\cdots,
\end{aligned}
$$

and the right-hand side of the amplitude equation (3.7), as

$$
A^{\prime}=\mu^{3} H+\cdots,
$$

where those terms depending on $C$ have been ignored, according to (A 1). Notice that the displayed term on the right-hand side of $(\Lambda 2)$ includes the three terms we are looking for. We insert (A 1) (A 2) into (2.1) (2.8) and set to zero the coefficients of $\mu^{2}$ and $\mu^{3}$. $\Lambda$ torder $\mu^{2}$ we obtain a non-singular linear problem whose solution is readily found as

$$
\begin{array}{ll}
u_{1}=u_{1}^{s}+\left(\mathrm{i} / 2 \omega_{0}\right) \partial q_{1}^{o} / \partial x, & v_{1}=v_{1}^{s}+\left(\mathrm{i} / 2 \omega_{0}\right) \partial q_{1}^{o} / \partial y, \\
q_{1}=q_{1}^{s}+q_{1}^{o}, & f_{1}=f_{1}^{s}+f_{1}^{o},
\end{array}
$$

where

$$
\begin{gathered}
q_{1}^{s}=\frac{\sigma^{2}-1}{\sigma^{2}} \omega_{0}^{2}\left(\left|A_{0}\right|^{2}+\left|B_{0}\right|^{2}\right), \quad f_{1}^{s}=\frac{\left(\sigma^{2}+1\right) \omega_{0}^{2}}{\sigma^{2}\left(1+4 T k^{2}\right)} A_{0} \bar{B}_{0} \mathrm{c}^{2 i k x}+\text { c.c., } \\
q_{1}^{o}=-\mathrm{c}^{2 i \omega_{0} t}\left[\frac{3 \sigma^{2}+1}{\sigma^{2}} \omega_{0}^{2} A_{0} B_{0}+\mathrm{i} \gamma_{1} \frac{\cosh [2 k(y+1)]}{\sinh 2 k}\left(A_{0}^{2} \mathrm{c}^{2 \mathrm{ik} k x}+B_{0}^{2} \mathrm{c}{ }^{2 i k x}\right)\right]+\text { c.c., } \\
f_{1}^{o}=\gamma_{2} \mathrm{e}^{2 i \omega_{0} t}\left(A_{0}^{2} \mathrm{e}^{2 \mathrm{i} k x}+B_{0}^{2} \mathrm{e}^{-2 i k x}\right)+\text { c.c., }
\end{gathered}
$$

with $\sigma=\tanh k$ as above, and

$$
\gamma_{1}=\frac{3 \omega_{0}\left[1-\sigma^{2}+\left(3-\sigma^{2}\right) T k^{2}\right]}{2 \sigma\left[\sigma^{2}+\left(\sigma^{2}-3\right) T k^{2}\right\rceil}, \quad \gamma_{2}=\frac{\left(3-\sigma^{2}\right) k\left(1+T k^{2}\right)}{2 \sigma\left[\sigma^{2}+\left(\sigma^{2}-3\right) T k^{2}\right\rceil} .
$$

Note that the superscripts $a$ and $o$ stand for the slowly varying and the short-timeoscillating parts.

Now, the linear problem at order $\mu^{3}$ is

$$
\begin{gathered}
u_{2 x}+v_{2 y}=0, \\
u_{3 t}+q_{3 x}=-\left[V_{0}\left(u_{1 y}^{s}-v_{1 x}^{s}\right) A_{0}+U_{0} H\right] \mathrm{e}^{\mathrm{i}\left(w_{0} t+\mathrm{i} k x\right.}+\mathrm{NRT}, \\
v_{3 i}+q_{3 y}=-\left[U_{0}\left(u_{1 y}^{\mathrm{s}}-v_{1 x}^{s}\right) A_{0}+V_{0} H\right] \mathrm{e}^{\mathrm{i}\left(\operatorname{lin}_{0} t+\mathrm{i} k x\right.}+\mathrm{NRT},
\end{gathered}
$$

in $x \in \mathbb{R},-1<y<0$, with boundary conditions

$$
\begin{gathered}
v_{3}=0 \text { al } y=-1, \\
v_{3}-f_{3 t}=\left[\left(\mathrm{i} k u_{1}^{s}-v_{1 y}^{s}+\mathrm{i} \gamma_{3}\left|A_{0}\right|^{2}+\mathrm{i} \gamma_{4}\left|B_{0}\right|^{2} A_{0}+H\right] \mathrm{e}^{\mathrm{i}\left(\xi_{0} t+\mathrm{i} k x\right.}+\mathrm{NRT},\right. \\
q_{3}-f_{3}+T f_{3 x x}=\left[\left(U_{0} u_{1}^{s}+V_{0} v_{1}^{s}+\gamma_{5}\left|A_{0}\right|^{2}+\gamma_{6}\left|B_{0}\right|^{2}\right) A_{0}-\omega_{0}^{2} \bar{B}_{0} \mathrm{e}^{2 \mathrm{i}\left(w_{0} i-\mathrm{i} k x\right.}+\mathrm{NRT},\right.
\end{gathered}
$$
at $y=0$, and

$$
u_{3}, v_{3}, q_{3} \text { and } f_{3} \text { periodic, of pcriod } L=2 m \pi / k \text {, in } x \text {, }
$$


where NRT stands for non-resonant terms (depending on $t$ as $\exp \left(\mathrm{ir}\left(\mathrm{o}_{0} t+\mathrm{i} s k x\right.\right.$ ), with $(r, s) \neq(1,1))$ and

$$
\begin{aligned}
& \gamma_{3}=-\left(3 k^{2} \omega_{0} \sigma+2 \gamma_{1} k^{2}\left(1+\sigma^{2}\right)+2 \gamma_{2} \omega k\right) /(2 \sigma), \\
& \gamma_{4}=\omega_{0} k^{3}\left[2 \sigma^{2}+1+\left(1+5 \sigma^{2}\right) T k^{2}\right\rfloor /\left[\sigma\left(1+4 T k^{2}\right)\right], \\
& \gamma_{5}=\left[8 k \sigma \omega_{0}^{2}+2 \gamma_{1} k\left(1-\sigma^{2}\right) \omega_{0}-2 \gamma_{2} \sigma^{2} \omega_{0}^{2}-3 k^{2}\left(1+T k^{2}\right) \sigma^{2}\right] /\left(2 \sigma^{2}\right), \\
& \gamma_{6}=-3 k^{2}\left(1+2 T k^{2}\right)-\left(1+\sigma^{2}\right) \omega_{0}^{4} /\left[\sigma^{2}\left(1+4 T k^{2}\right)\right]
\end{aligned}
$$

with $\gamma_{1}$ and $\gamma_{2}$ as given by (A7) and $\sigma=\tanh k$, as above. Now $H$ is readily calculated by requiring the solution of the $O\left(\mu^{3}\right)$ problem above to be bounded in the short-time-scale $t \sim 1$. That solvability condition is readily applied through the equation that results when (A 9) and (A 10) are multiplied by $U_{0} \exp \left(-\mathrm{i} \omega_{0} t-\mathrm{i} k x\right)$ and $\bar{V}_{0} \exp \left(-\mathrm{i} \omega_{0} l-\mathrm{i} k x\right)$, respectively, the resulting equations are added and integrated in $0<x<L,-1<y<0$, integration by parts is applied, and the continuity equation, the boundary conditions and equations (3.6) are taken into account. After some algebra, we obtain

$$
H=\mathrm{i}\left(\varkappa_{3}\left|A_{0}\right|^{2}-\alpha_{4}\left|B_{0}\right|^{2}\right) A_{0}+\mathrm{i} \alpha_{5} \bar{B}_{0} \mathrm{e}^{2 \mathrm{i}\left(\omega-\omega_{0}\right) t}-\mathrm{i} \alpha_{6} \int_{0}^{L} \int_{-1}^{o} g(y) u_{1}^{s}(x, y, t) \mathrm{d} x \mathrm{~d} y A_{0},
$$

where $x_{3}, \ldots, x_{6}$ and $g$ are as given in equations (3.10)-(3.11), in $\$ 3$. Finally, we need only replace $\left(A_{0}, B_{0}\right)$ by $(A, B) \mathrm{e}^{\mathrm{i}\left(\omega, \omega \omega_{0}\right) t}$ to obtain $(3.7)(3.8)$.

\section{RFFHRFNCFS}

Batchelor, G. K. 1967 An Introduction to Fhid Dynamics. Cambridge University Press.

Canlto, C., Hlssaini, H. Y., Quarteroni, K. \& Zang, T. A. 1988 Spectral Methods in Fluid Dynamics. Springer.

Crawford, J. D., KNobloch, E. \& Riecke, H. 1990 Period-doubling mode interations with circular symmetry. Physica D 44, 340-396.

Cross, M. C. \& Hohenberg, P. C. 1993 Pattern formation oulside of cquilibrium. Rev. Mod. Phys. 65, 851-1112.

Davgelmayr, G., Hettel, J. \& KNOBloch, E. 1997 Parity-brcaking bifurcation in inhomogencous systcms. Nonlinearity 10, 1093-1114.

DAvi:y, A. \& Siliwarison, S. 1974 On three-dimensional packets of surface waves. Proc. R. Soc. Lond. A 338, 101-110.

Dousuy, S. 1990 Experimental study of the Faraday instability. J. Fluid Mech. 221, 383409.

Dounajy, S., FAuvi, S. \& Tiluni, O. 1989 Oscillatory phase modulation of parametrically forced surface waves. Europhys. Lett. 10,309 315.

FARADAY, M. 1831 On the forms and states assumed by fluids in contact with vibrating elastic surfaces. Phil. Trans. R. Soc. Iond. 121, 319340.

Fauve, S., Dolady, S. \& Thual, O. 1991 Drift instabilities in cellular patterns. J. Phys. II 1 , 311322.

GreEne, J. M. \& KIM, J. S. 1988 The steady states of the Kuramoto-Sivashinsky equation. Physica I) 33,99120 .

IlANSEN, P. L. \& ALstrom, P. 1997 Perturbation theory of parametrically driven capillary waves at low viscosity. J. Fhid Mech. 351, 301-344.

I Ienderson, D. M. \& Miles, J. W. 1994 Surface-wave damping in a circular cylinder with a fixed contact line. J. Fluid Mech. 275, 285-299.

Iliglera, M., Nicolás, J. A. \& Vega, J. M. 1994 Linear oscillations of weakly dissipative axisymmetric liquid bridges. Phys. Fluids A 6, 438-450.

Higlera, M. \& Nicolás, J. A. 1997 Lincar non-axisymmetric oscillations of ncarly-inviscid liquid bridges. Phys. Fluids 9, 276-285. 
Iskandaravi, M. \& Lic, P. L. F. 1991 Mass transport in threc-dimensional water waves. J. Fluid Mech. 231, 417-437.

Kiyashko, S. V., Korzinov, L. N., Rabinovich, M. I. \& Tsimring, L. S. 1996 Rotating spirals in at Faraday expcriment. Phys. Rev. E 54, 5037-5040.

LАмв, H. 1932 Hydrodynamcis. Cambridge University l'ress.

Li:Ilsovicir, S. \& PAorucer, S. 1981 The instability of the ocean to Langmuir circulations. J. Fluid Mech. 102, 141167.

LıU, A. K. \& DAvis, S. H. 1977 Viscous attenuation of mean drift in water waves. J. Fluid Mech. 81,6384

Longuet-IIIgGins, M. S. 1953 Mass transport in water waves. Phil. Trans. R. Soc. A 245, 535-581.

Martel, C. \& KNobloch, E. 1997 Damping of nearly-inviscid water waves. Phys. Rev. E 56, $5544-5548$.

Martel, C.. Nicolés, J. A. \& VEGA, J. M. 1998 Surface-wave damping in a brimful circular cylinder. J. Filuid Mech. 360, 213-228. See also Corrigendum, 373, 379.

Mashayek, F. \& Ashgriz, N. 1998 Nonlincar oscillations of drops with internal circulation. Phys. Ihids 10, 1071-1082.

Miles, J. W. 1993 On Faraday waves. J. Fluid Mech. 248, 671-683.

Miles, J. \& Henderson; D. 1990 Parametrically lorced surface waves. Amm. Rev. Fluid Mech. 22, $143-165$.

MıIiWski, P. A. \& Bı:Nivi, D. J. 1995 Resonant interactions between vortical flows and water waves. Part I: Decp watcr. Stud. Appl. Maths 94, 131-167.

Minilk, S. T. 1991 Square patterns and secondary instabilities in driven capillary waves. J. Fluid Mech. 225, 81-100.

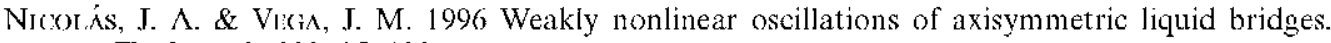
J. Fluid Mech. 328, 95-128.

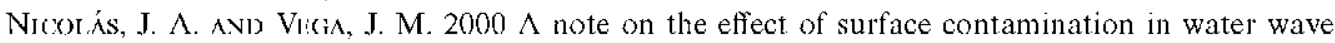
damping. J. Fluid Mech. 410, 367373.

Ruli:, M. 2001 Steady streaming. Annu. Rev. Fluid Mech. 33, 4365.

SchlichtING, II. 1932 Berechnung ebener periodischer Grenzschichtströmungen. Phys. Z. 33, 327335.

Thual, O., Douady, S. \& Fauve, S. 1989 Parametric instabilities. In Insiabilities and Nonequilibrium Structures II (ed. E. Tirapegui \& D. Villaroel), pp. 227-237. Kluwer.

VEGA, J. M., KVOBLOCH, E. \& MARTEL, C. 2001 Nearly inviscid laraday waves in annular containers of moderately large aspect ratio. Physica D 154, 147-171.

YaN, B., INGHam, D. B. \& Morton, B. R. 1993 Streaming flow induced by an oscillating cascade of circular cylinders. J. Fhid Mech. 252, 313-336. 